\title{
Teoria métrica de curvas reais e complexas'
}

\author{
Alexandre César Gurgel Fernandes
}

Orientadora: Profa. Dra. Maria Aparecida Soares Ruas

Tese apresentada ao Instituto de Ciências Matemáticas e de Computação - ICMC-USP. como parte dos requisitos para obtenção do título de Doutor em Ciências - Matemática.

\section{USP - São Carlos}

Dezembro de 2001

${ }^{1}$ Este trabalho foi completamente financiado pelo $\mathrm{CNPq}$ 


\section{A Comissão Julgadora:}

Profa. Dra. Maria.Aparecida Soares Ruas

Prof. Dr. Abramo Hefez

Prof. Dr. Marcio Gomes Soares

Prof. Dr. Lev Birbrair

Prof. Dr. Marcelo José Saia

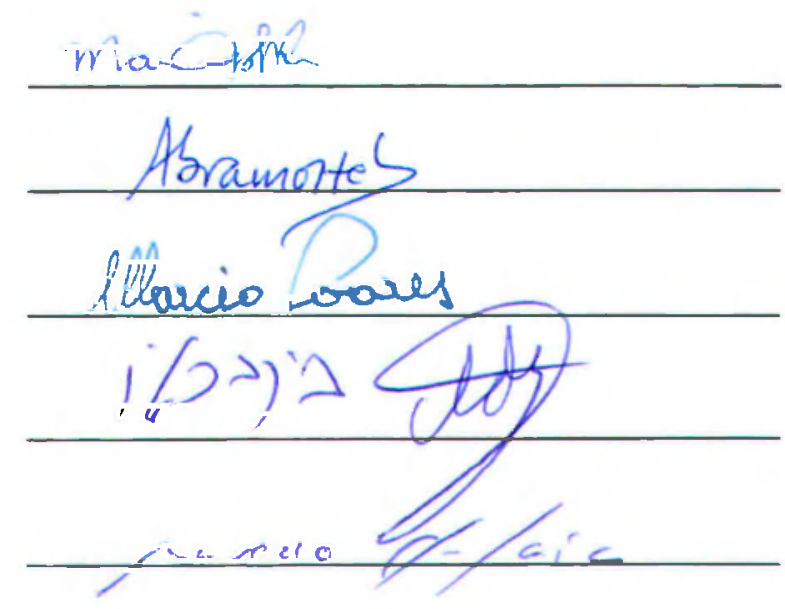


Aos meus pais,

Silvio e Lenir. 


\section{Agradecimentos}

A Deus pelas infinitas bênçãos recebidas durante a realização deste trabalho inclusive a graça de ser papai.

Aos meus pais, Sílvio e Lenir, às minhas irmãs, Alessandra, Aline e Amanda por estarem do meu lado em todas as situações difíceis que enfrentei.

$\grave{A}$ minha esposa, Claudia, pelo interese neste projeto, pelas observações a respeito de minha postura profissional, que foram fundamentais neste trabalho e pelas inúmeras vezes em que se dispôs a ficar algumas horas da madrugada ouvindo-me falar de resultados obtidos (a maioria incorretos).

À minha professora e orientadora Dra. Maria Aparecida Soares Ruas por me introduzir com bastante mestria e de forma muito rápida na Teoria de Singularidades, por várias leituras e correções deste trabalho e pelo trabalho de orientação.

Ao professor Dr. Lev Birbrair por me incentivar a estudar Teoria métrica de singularidades, dividir comigo a sua enorme experiência neste assunto e, finalmente, por ter me acompanhado até hoje em se tratando de amizade e profissão.

Ao ICMC e ao Grupo de Singularidades pelo excelente suporte técnico dado.

Seja louvado o nome de Jesus!!! 


\section{Resumo}

Neste trabalho abordamos o problema de classificação de conjuntos singulares sob o ponto de vista métrico. Como resultado principal, apresentamos um teorema de classificaçāo de germes de curvas complexas, munidos da métrica euclidiana induzida, módulo homeomorfismos bi-Lipschitz. A estratégia usada para a obtenção deste resultado foi o estudo do contato de arcos reais convenientes nessas curvas. 


\section{Abstract}

In this work we approach the problem of classification of singular sets under the metric viewpoint. As the main result, we present a theorem of classification of germs of complex curves, equipped with the induced Euclidean metric, module bi-Lipschitz homeomorphisms. The used strategy for the attainment of this result was the study of the contact of convenient real arcs in these curves. 


\section{Sumário}

Introdução

1 Preliminares 4

1.1 Conjuntos e aplicações semi-algébricas . . . . . . . . . . . 4

1.2 Conjuntos e aplicaçōes subanalíticas . . . . . . . . . . 5

1.3 Subespaços euclidianos, aplicações bi-Lipschitz e o Teorema de Kirszbraun 6

1.4 Expoentes característicos de Puiseux f . . . . . . . . . . . . 7

1.5 Projeçōes gerais . . . . . . . . . . . . . . . . 9

2 Teoria métrica de curvas reais $\quad 10$

2.1 Semicomplexo de Hölder . . . . . . . . . . . . . . . . . 11

2.2 Sobre o tipo Lipschitz de curvas reais . . . . . . . . . . . . . . . . 13

2.3 Sobre famílias de curvas reais f . . . . . . . . . . . . . . . 17

2.4 Sobre famílias de curvas reais quase-homogêneas . . . . . . . . . . 25

3 Teoria métrica de curvas complexas 29

3.1 Arcos de Teste . . . . . . . . . . . . . . . . . 31

3.2 Caso irredutível . . . . . . . . . . . . . . . . . . 32

3.3 Caso redutível . . . . . . . . . . . . . . . . . . . . 41

3.4 Tipo bi-Lipschitz de curvas complexas . . . . . . . . . . . . . 44

4 bi-Lipschitz trivialidade de funções $\quad 48$

4.1 Um resultado do tipo Kuo . . . . . . . . . . . . . . . . . 50 


\section{Introdução}

A partir de resultados de S. Lojasiewicz sobre propriedades métricas de conjuntos semi-analíticos, desenvolveram-se alguns trabalhos, iniciados por S. Lojasiewicz e seus alunos, tendo como objetivo principal o estudo de conjuntos singulares sob o ponto de vista métrico, originando então o que hoje é conhecida como teoria métrica de singularidades. Como referência mais recente, essa teoria tem como autores mais ativos, T Mostowski, A. Parusinski, L. Birbrair, K Kurdyka, P. Orro, Y. Yomdim, dentre outros.

Em [2], L. Birbrair classifica completamente os germes de superfícies semi-algébricas (singulares), munidos da métrica geodésica intrínseca, módulo homeomorfismos biLipschitz. Nesta direção, surge naturalmente a pergunta: como classificar os germes de superfícies (singulares), munidos da métrica euclidiana induzida, módulo homeomorfismos bi-Lipschitz?

Uma estratégia para responder à pergunta acima seria a redução do problema de classificação de superfícies ao problema de classificação de curvas, isto é, estamos apostando na existência de curvas "características"que detectem completamente o comportamento métrico dessas superfícies. Aqui, resolvemos o problema de classificação de curvas complexas (caso bem particular de superfícies reais) usando essa estratégia. Claramente, esta abordagem, diante da pergunta inicial, exige uma análise acurada dos germes de curvas a qual fazemos no Capítulo 2. Mais especificamente, neste capítulo, classificamos completamente o "tipo bi-Lipschitz" de curvas e, como conseqüência dessa classificação, obtemos resultados de caracterização de famílias de curvas bi-Lipschitz triviais.

O Capítulo 3 contém os principais resultados de [11]. Aqui, apresentamos uma 
classificação completa dos germes de curvas complexas planas, munidos da métrica euclidiana induzida, módulo homeomorfismos bi-Lipschitz. Ainda neste capítulo, apresentamos uma rápida discussão a respeito de curvas espaciais.

No Capítulo 4, introduzimos, rapidamente, o problema da $\mathcal{R}$-bi-Lipschitz equivalência de germes de aplicações e apresentamos um teorema de bi-Lipschitz determinação para a classe dos germes com singularidade isolada. 


\section{Capítulo 1}

\section{Preliminares}

Sejam $A$ um subconjunto de $\mathbb{R}^{n}, a \in A$ e sejam $f, g:(A, a) \rightarrow \mathbb{R}, 0$ germes de funçōes não negativas. Denotamos $f \lesssim g$ quando existe uma vizinhaça $U$ de $a$ em $\mathbb{R}^{n}$ e uma constante $K$ tal que $f(x) \leqslant K g(x), \forall x \in A$. Denotamos $f \approx g$ quando ocorre $f \lesssim g$ e $g \lesssim f$. Finalmente, denotamos

$$
f \ll g \quad \text { quando } \quad \lim _{x \rightarrow a} \frac{f(x)}{g(x)}=0 \quad \text { e } \quad f \gg g \quad \text { quando } \quad \lim _{x \rightarrow a} \frac{g(x)}{f(x)}=0 .
$$

\subsection{Conjuntos e aplicações semi-algébricas}

Dizemos que um subconjunto $A \subset \mathbb{R}^{n}$ é semi-algébrico (semi-analítico) se existem polinômios (funções analíticas) $f_{i j}, g_{i j}: \mathbb{R}^{n} \rightarrow \mathbb{R}, 1 \leqslant i \leqslant p, 1 \leqslant j \leqslant q$ tais que

$$
A=\bigcup_{i=1}^{p}\left\{x \in \mathbb{R}^{n}: f_{i j}=0, g_{i j}>0,1 \leqslant j \leqslant q\right\} .
$$

Um fato, que não é óbvio, é que se $A \subset \mathbb{R}^{n}$ é semi-algébrico, então existem subvariedades $A_{1}, \ldots, A_{m}$ de $\mathbb{R}^{n}$, conexas, semi-algébricas tais que $A=\cup_{i} A_{i}$ ([5]). Definimos a dimensão de $A$ como o máximo das dimensões das variedades $A_{1}, \ldots, A_{m}$.

Seja $A \subset \mathbb{R}^{n}$ semi-algébrico. Dizemos que uma aplicação $F: A \rightarrow \mathbb{R}^{k}$ é semialgébrica se o seu gráfico é um subconjunto semi-algébrico de $\mathbb{R}^{n} \times \mathbb{R}^{k}$.

Teorema 1.1.1. (Tarski-Seidenberg) Seja $\Pi: \mathbb{R}^{n} \times \mathbb{R}^{m} \rightarrow \mathbb{R}^{m}$ a projeção canônica. Se $A \subset \mathbb{R}^{n} \times \mathbb{R}^{m}$ é semi-algébrico, então $\Pi(A)$ é semi-algébrico. 
O toerema acima permite afirmar que a classe dos conjuntos semi-algébricos é estável por operaçōes booleanas e aplicações semi-algébricas. Quanto à classe dos conjuntos semi-analíticos, não podemos afirmar o mesmo, por conta disso se faz necessário o estudo sobre conjuntos subanalíticos.

\subsection{Conjuntos e aplicações subanalíticas}

As definições e resultados destacados nesta seção podem ser encontrados em [6]. Seja $X$ um espaço analítico real. Um subconjunto $A \subset X$ é dito subanalítico em $x \in X$, se existe uma vizinhança $U$ de $x$ em $X$ e uma família $\left\{f_{i j}: 1 \leqslant i \leqslant k, j=1,2\right\}$ de morfismos analíticos próprios: $Y_{i j} \rightarrow X_{l_{U}}$, em que $Y_{i j}$ é espaço analítico real, tais que

$$
A \cap U=\bigcup_{i=1}^{k}\left(\operatorname{Im}\left(\mathrm{f}_{\mathrm{i} 1}\right)-\operatorname{Im}\left(\mathrm{f}_{\mathrm{i} 2}\right)\right) .
$$

Dizemos que $A$ é um subconjunto subanalítico em $X$ quando $A$ é subanalítico em cada ponto de $X$.

A classe dos conjuntos subanalíticos é a menor classe de subconjuntos de espaços analíticos contendo os conjuntos semi-analíticos e estável por morfismos próprios e operações booleanas.

Teorema 1.2.1. Sejam $X$ e $Y$ dois espaços analíticos reais, $\Pi: X \times Y \rightarrow X a$ projeção canônica. Se $V$ é um subconjunto compacto subanalítico em $Y$, e $A \subset X \times V$ um subconjunto subanalítico em $X \times Y$, então $\Pi(A)$ é subanalítico em $X$.

Esse teorema é o análogo do Tarski-Seidenberg para o caso subanalítico.

Sejam $K, L$ subconjuntos subanalíticos respectivamente nos espaços analíticos $X, Y$. Dizemos que uma aplicação contínua $F: K \rightarrow L$ é subanalítica se o seu gráfico $\operatorname{Graf}(\boldsymbol{f})$ é um subconjunto subanalítico em $X \times Y$.

O Lema a seguir é o que chamamos de Decomposição de Puiseux de uma função subanalítica.

Lema 1.2.2. Seja $f:[0,1] \rightarrow \mathbb{R}$ uma função subanalítica tal que $f(0)=0$ e $f(t) \neq 0$ para cada $t \neq 0$ numa vizinhança de 0 . Então, existe um racional positivo $\alpha$ e uma 
função subanalítica $h:[0, \epsilon) \rightarrow \mathbb{R}$ tais que $h(0) \neq 0$ e $f(t)=t^{\alpha} h(t)$ para cada $t \in[0, \epsilon)$.

\subsection{Subespaços euclidianos, aplicações bi-Lipschitz e o Teorema de Kirszbraun}

Nesta tese, salvo menção em contrário, consideramos $\mathbb{R}^{n}$ munido da métrica euclidiana e entendemos por subespaço euclidiano os subconjuntos de $\mathbb{R}^{n}$ munidos da métrica induzida.

Sejam $\left(X_{1}, d_{1}\right)$ e $\left(X_{2}, d_{2}\right)$ espaços métricos. Uma aplicação $F: X_{1} \rightarrow X_{2}$ é chamada Lipschitz se existe um número $c>0$ tal que

$$
d_{2}(F(x), F(y)) \leqslant c d_{1}(x, y), \quad \forall x, y \in X_{1}
$$

Nesse caso dizemos também que $F$ é $c$-Lipschitz. Dizemos que $F$ é bi-Lipschitz quando $F$ é Lipschitz e possui uma inversa Lipschitz.

A definição de homeomorfismo bi-Lipschitz acima é uma relação de equivalência sobre os subespaços de $\mathbb{R}^{n}$. Sobre esses mesmos subespaços, temos naturalmente outra relaçāo, a qual dizemos que detecta o tipo bi-Lipschitz dos subespaços de $\mathbb{R}^{n}$. De fato, sejam $X_{1}$ e $X_{2}$ subespaços de $\mathbb{R}^{n}$, então $X_{1}$ possui o mesmo tipo bi-Lipschitz de $X_{2}$ se existe um homeomorfismo bi-Lipschitz $\phi: \mathbb{R}^{n} \rightarrow \mathbb{R}^{n}$ tal que $\phi\left(X_{1}\right)=X_{2}$.

Observação 1.3.1. Como nos interessamos em classificar subespaços euclidianos módulo homeomorfismos bi-Lipschitz, nāo fazemos distinçāo entre a métrica euclidiana e qualquer outra métrica equivalente a esta.

O Teorema de Kirsbraun considera o problema de extensão de funções Lipschitz. Kirsbraun, como caso particular de um resultado sobre extensāo de contrações de um espaço vetorial $V \rightarrow \mathbb{R}$, obteve uma prova para o fato que aplicações $c$-Lipschitz $Y \rightarrow \mathbb{R}$, em que $Y$ é subespaço métrico de $X$, se estendem a aplicações $c$-Lipschitz $X \rightarrow \mathbb{R}$. Esta prova é baseada em métodos de indução transfinita. Em 1934, E. McShane obtém uma nova prova para esse fato, sugerindo uma fórmula explícita 
para a extensão (cf: [20]). A seguir, apresentamos uma versão fraca do Teorema de Kirszbraun, já enunciado com a fórmula de McShane(cf. [10])

Teorema 1.3.2. Sejam $X \subset \mathbb{R}^{n}$ um subespaço euclidiano e $f: X \rightarrow \mathbb{R}$ uma aplicação c-Lipschitz. Entāo,

$$
F(z)=\sup _{x \in X}(f(x)-c|x-z|), \quad z \in \mathbb{R}^{n}
$$

é uma extensäo c-Lipschitz de $f$.

Segue-se que se $f$ depende continuamente de alguns parâmetros $u=\left(u_{1}, \ldots, u_{p}\right)$; isto é, $f(x, u), x \in X$, é contínua como função das variáveis $(x, u)$ e é $c$-Lipschitz com respeito a $x \operatorname{com} c$ independente de $u$, então $f$ possui uma extensão $c$-Lipschitz $F(x, u), x \in \mathbb{R}^{n}$. Agora, se $v$ é um campo de vetores $c$-Lipschitz definido em $X$ e dependendo continuamente de alguns parâmetros $u$, então podemos aplicar o Teorema de Kirszbraun para cada uma das funçōes coordenadas $v$ e obter uma extensão $c \sqrt{n}$ Lipschitz $V$ de $v$ a $\mathbb{R}^{n}$. Essa construção pode ser encontrada em [23].

Observaçāo 1.3.3. A fórmula de McShane, apresentada no teorema acima, é muito bem-vinda, não só por apresentar uma nova prova para o Teorema de Kirszbraun. Com efeito, a partir de (1.1), podemos concluir que se $f$ é semi-algébrica (subanalítica), então a sua extensão $F$ vem semi-algébrica (subanalítica).

A seguinte proposicão, provada em [12], justifica a nossa escolha por trabalhar, no capítulo que trata da teoria métrica de curvas complexas, com homeomorfismos subanalíticos bi-Lipschitz e não apenas homeomorfismos bi-Lipschitz.

Proposição 1.3.4. Seja $h:\left(\mathbb{R}^{n}, 0\right) \rightarrow\left(\mathbb{R}^{n}, 0\right)$ homeomorfismo subanalítico. Então, $h$ é bi-Lipschitz se, e somente se, para cada par de curvas analíticas $\alpha_{1}, \alpha_{2}:[0, \delta) \rightarrow$ $\left(\mathbb{R}^{n}, 0\right)$,

$$
\operatorname{ord}_{t}\left\|h\left(\alpha_{1}(t)\right)-h\left(\alpha_{2}(t)\right)\right\|=\operatorname{ord}_{t}\left\|\alpha_{1}(t)-\alpha_{2}(t)\right\|
$$

\subsection{Expoentes característicos de Puiseux}

Seja $(C, 0)$ um germe de curva analiticamente irredutível em $\mathbb{C}^{2}($ Ramo), a menos de uma mudança analítica de coordenadas, podemos supor que $(C, 0)$ tem uma 
parametrização do tipo abaixo:

$$
\begin{aligned}
& x=t^{m} \\
& y=t^{n}+a_{2} t^{n_{2}}+\ldots
\end{aligned}
$$

em que $m$ é a multiplicidade de $(C, 0), m$ nāo divide o inteiro $n$ e $y(t) \in \mathbb{C}\{t\}$. A série de potências fracionárias $y\left(x^{\frac{1}{m}}\right)$ é conhecida como Parametrização de NewtonPuiseux de $(C, 0)$ e todas as outras parametrizações de Newton-Puiseux de $(C, 0)$ são obtidas da parametrização acima via $x^{\frac{1}{m}} \mapsto w x^{\frac{1}{m}}$ em que $w$ é raiz $m$-ésima da unidade. Observo que estamos admitindo que 0 seja uma singularidade de $C$

Denotamos $\beta_{0}=m$ e $\beta_{1}=n$. Seja $e_{1}=\left(\beta_{1}, \beta_{0}\right)$ o máximo divisor comum desses dois inteiros. Agora, definimos $\beta_{2}$ como o menor expoente aparecendo em $y(t)$ que nāo é divisível por $e_{1}$. Definimos $e_{2}=\left(e_{1}, \beta_{2}\right)$; temos $e_{2}<e_{1}$, e continuamos esse processo. Supondo já definido $e_{i}=\left(e_{i-1}, \beta_{i}\right)$, definimos $\beta_{i+1}$ como o menor expoente aparecendo em $y(t)$ que não é divisível por $e_{i}$. Como a seqüência de inteiros positivos

$$
m>e_{1}>\cdots>e_{i}>\cdots
$$

é estritamente decrescente, existe um inteiro $g$ tal que $e_{g}=1$. De posse desses dados, podemos reescrever (1.2) da seguinte maneira:

$$
\begin{aligned}
x & =t^{m} \\
y & =t^{\beta_{1}}+a_{\beta_{1}+e_{1}} t^{\beta_{1}+e_{1}}+\ldots+a_{\beta_{1}+k_{1} e_{1}} t^{\beta_{1}+k_{1} e_{1}} \\
& +a_{\beta_{2}} t^{\beta_{2}}+a_{\beta_{2}+e_{2}} t^{\beta_{2}+e_{2}}+\cdots+a_{\beta_{q}} t^{\beta_{q}}+a_{\beta_{q}+e_{q}} t^{\beta_{q} e_{q}}+\cdots \\
& +a_{\beta_{g}} t^{\beta_{g}}+a_{\beta_{g}+1} t^{\beta_{g}+1}+\cdots
\end{aligned}
$$

tal que, por construção os coeficientes de $t^{\beta_{i}} ; i \geqslant 1$ são distintos de zero. Definimos os inteiros $m_{i}$ e $n_{i}$ pelas igualdades:

$$
\begin{aligned}
e_{i-1} & =n_{i} e_{i} \\
\beta_{i} & =m_{i} e_{i} \quad \text { para } \quad 1 \leqslant i \leqslant g
\end{aligned}
$$

e observamos que podemos reescrever a expansão de $y$ em potências fracionárias de $x$ da seguinte forma:

$$
y\left(x^{\frac{1}{m}}\right)=a_{\beta_{1}} x^{\frac{m_{1}}{n_{1}}}+a_{\beta_{1}+e_{1}} x^{\frac{m_{1}+1}{n_{1}}}+\cdots+a_{\beta_{1}+k_{1} e_{2}} x^{\frac{m_{1}+k_{1}}{n_{1}}}
$$




$$
\begin{aligned}
& +a_{\beta_{2}} x^{\frac{m_{2}}{n_{1} n_{2}}}+a_{\beta_{2}+e_{2}} x^{\frac{m_{2}+1}{n_{1} n_{2}}}+\cdots+a_{\beta_{q}} x^{\frac{m_{q}}{n_{1} n_{2} \cdots n_{q}}}+a_{\beta_{q}+e_{q}} x^{\frac{m_{q}+1}{n_{1} n_{2} \cdots n_{q}}}+\cdots \\
& +a_{\beta_{g}} x^{\frac{m_{g}}{n_{1} n_{2} \cdots n_{g}}}+a_{\beta_{g}+1} x^{\frac{m_{g}+1}{n_{1} n_{2} \cdots n_{g}}}+\cdots
\end{aligned}
$$

A seqüência de inteiros $\beta(C)=\left(\beta_{0}, \beta_{1}, \ldots, \beta_{g}\right)$ é conhecida como a seqüência de expoentes característicos de $C$, e a seqüência $\left(m_{1}, n_{1}\right) \ldots\left(m_{g}, n_{g}\right)$ é conhecida como a seqüência de pares característicos de $C$.

\subsection{Projeções gerais}

Seja $(C, 0)$ um germe de curva analítica espacial (reduzida) definida por um ideal $I \subset \mathbb{C}\left\{x_{1}, \ldots, x_{d}\right\}$. Consideremos uma projeção linear $p: \mathbb{C}^{d} \rightarrow \mathbb{C}^{2}$. Seja. $M$ o espaço de todas as projeções, pensado como um subespaço do espaço das matrizes $d \times 2$. Equipemos $M$ com a topologia complexa (ou de Zariski). A projeção linear $p$ é dita uma projeção plana geral para $C$ em 0 , se para qualquer seqüência de pares $\left(a_{i}, b_{i}\right) \in(\mathbb{C} \backslash 0) \times(\mathbb{C} \backslash 0)$ tendendo a $(0,0)$, a direção limite das retas secantes $\overline{a_{i} b_{i}}$ (para qualquer subseqüência) não está contido no núcleo de $p$.

Observação 1.5.1. Em [28], B. Teissier observa que projeções gerais para $C$ em 0 , ocorrem genericamente. Além disso, B. Teissier prova que $p(C)$ define um germe de curva analítica plana (reduzida) e $p$, quando restrita a $C$, define um germe de homeomorfismo bi-Lipschitz. 


\section{Capítulo 2}

\section{Teoria métrica de curvas reais}

O seguinte exemplo

$$
X_{t}=\left\{(x, y) \in \mathbb{R}^{2}: x y(x-y)(x-t y)=0\right\} \quad t>1
$$

devido a H. Whitney (1965), motivou autores como D.Trotman, B. Teissier, T.C. Kuo, T. Mostowski e muitos outros a considerar o estudo de conjuntos singulares módulo relações de equivalências mais fracas do que diferenciáveis e mais fortes do que topológicas, pois para cada par de números reais $t>s>1$ temos que $X_{t}$ não é $C^{1}$ equivalente a $X_{s}$, isto é, nāo existe um difeomorfismo $C^{1} \phi:\left(\mathbb{R}^{2}, 0\right) \rightarrow\left(\mathbb{R}^{2}, 0\right)$ tal que $\phi\left(X_{t}\right)=\left(X_{s}\right)$. Este fenômeno indica uma grande rigidez no problema de classificação do ponto de vista diferenciável. Por outro lado, a família

$$
C_{m}=\left\{(x, y) \in \mathbb{R}^{2}: y^{2 m+1}=x^{2}\right\}, \quad m \in \mathbb{N}
$$

mostra quão flexível é o problema de classificaçāo topológica de conjuntos singulares. Com efeito, para cada par de inteiros positivos $m \neq n$ temos um homeomorfismo $\phi:\left(\mathbb{R}^{2}, 0\right) \rightarrow\left(\mathbb{R}^{2}, 0\right)$ tal que $\phi\left(C_{m}\right)=C_{n}$.

De forma nada surpreendente, mostramos que a família (2.1) define uma única classe módulo uma relação bi-Lipschitz, isto é, para cada par de números reais $t>s>$ 1 temos um homeomorfismo bi-Lipschitz $\phi:\left(\mathbb{R}^{2}, 0\right) \rightarrow\left(\mathbb{R}^{2}, 0\right)$ tal que $\phi\left(X_{t}\right)=X_{s}$. Isto indica que, comparada à relação diferenciável, a relação métrica acima não é tão rígida. Por outro lado, a família (2.2) tem seus elementos dois a dois não 
equivalentes módulo a relação métrica acima. T.Mostowski [22], A. Parusinski [24], L. Birbrair [2] são alguns nomes que consideram o problema de classificação métrica de singularidades.

Este capítulo é destinado exclusivamente ao estudo de conjuntos singulares de dimensão real 1, vistos como subespaços euclidianos, módulo homeomorfismos biLipschitz. De forma superficial, subdividimos este capítulo da seguine forma. $\mathrm{Na}$ primeira seção deste capítulo, listamos alguns resultados de classificação de curvas reais os quais estão no artigo [3]. Observo que as idéias apresentadas nesta seção são fundamentais para o desenvolvimento desta tese. Na segunda seção, resolvemos o problema de caracterizaçāo do tipo bi-Lipschitz de curvas reais demonstrando que o semicomplexo de Hölder (cf. [3]) é um invariante completo para esse problema. $\mathrm{Na}$ terceira seção, apresentamos um teorema de caracterização de famílias de curvas bi-Lipschitz triviais. Ainda na terceira seção, abordamos o problema de trivialização bi-Lipschitz de famílias de curvas semi-algébricas por desdobramentos bi-Lipschitz semi-algébricos e, nessa direção, obtemos um teorema que carateriza completamente famílias de curvas planas por tais desdobramentos. Na quarta seção, aplicamos os resultados da seção anterior para o estudo de famílias quase-homogêneas.

\subsection{Semicomplexo de Hölder}

Os resultados apresentados nesta seção, exceto a Proposição 2,1.2, estão publicados em [3].

Seja $(X, x)$ um germe de subconjunto semi-algébrico em $\mathbb{R}^{p}$, de dimensão reàl 1, com semi-ramos $X=\bigcup_{i \in I} X_{i}$. Definimos para cada par de semi-ramos $X_{i}, X_{j}$, o seguinte número:

$$
\operatorname{sh}\left(X_{i}, X_{j}\right)=\operatorname{ord}_{r}\left[\operatorname{dist}\left(X_{i} \cap S_{\tau}(x), X_{j} \cap S_{\tau}(x)\right)\right]
$$

em que $S_{r}(x)$ denota a esfera $\left\{y \in \mathbb{R}^{p}:\|x-y\|=r\right\}$.

Lema 2.1.1. $\operatorname{sh}\left(X_{i}, X_{j}\right)=\operatorname{ord}_{r}\left[\operatorname{dist}\left(X_{i}-B_{r}(x), X_{j}-B_{r}(x)\right)\right], \forall i \neq j$, em que $B_{r}(x)$ denota a bola aberta $\left\{y \in \mathbb{R}^{p}:\|x-y\|<r\right\}$. 
A proposição abaixo está provada na primeira seção do Capítulo 3 .

Proposição 2.1.2. Seja $F:(X, 0) \rightarrow \mathbb{R}^{p}$ o germe de uma aplicação semi-algébrica tal que sua restrição a cada $X_{i}$ é Lipschitz. Denotemos por $x_{i}(r)$ o ponto na interseção $X_{i} \cap S_{r}(0) . S e$

$$
\| F\left(x_{i}(r)\right)-F\left(x_{j}(r)\|\lesssim\| x_{i}(r)-x_{j}(r) \|, \forall i, j,\right.
$$

então $F$ é o germe de uma aplicação Lipschitz.

Teorema 2.1.3. Sejam $(X, x)$ e $(Y, y)$ germes de subconjuntos semi-algébricos, de dimensão real 1 , equipados com a métrica euclidiana induzida e com semi-ramos $X=\bigcup_{i \in I} X_{i}$ e $\underline{Y}_{1}=\bigcup_{i \in J} Y_{j}$. Então, existe $F:(X, x) \rightarrow(Y, y)$ bi-Lipschitz se, $e$ somente se, existe uma bijeção $\phi \vdots I \rightarrow J$ tal que $\operatorname{sh}\left(X_{i}, X_{j}\right)=\operatorname{sh}\left(Y_{\phi(i)}, Y_{\phi(j)}\right)$, para cada par $i \neq j \in I$.

Prova. Sem perda de generalidade, podemos supor $x=0=y$. Suponhamos que $F:(X, 0) \rightarrow(Y, 0)$ seja uma aplicação bi-Lipschitz. Sejam $0<c_{1} \leqslant c_{2}$ constantes de Lipschitz de $F$. Como $F$ é um homeomorfismo, temos que $I$ e $J$ possuem a mesma quantidade de elementos e para cada $i \in I$ existe um único $\phi(i) \in J$ tal que $\left(F\left(X_{i}\right), 0\right)=\left(Y_{\phi(i)}, 0\right)$. Isto é, $\phi: I \rightarrow J$ definida como acima é uma bijeção.

Sejam $m=\operatorname{sh}\left(X_{i}, X_{j}\right)$ e $n=\operatorname{sh}\left(Y_{\phi(i)}, Y_{\phi(j)}\right)$. Consideremos também $r^{m} h(r)$ e $r^{n} g(r)$ as decomposiçōes de Puiseux de

$$
\operatorname{ord}_{r}\left[\operatorname{dist}\left(X_{i}-B_{r}(0), X_{j}-B_{r}(0)\right)\right] \quad \text { e } \quad \operatorname{ord}_{r}\left[\operatorname{dist}\left(Y_{\phi(i)}-B_{r}(0), Y_{\phi(j)}-B_{\tau}(0)\right)\right]
$$

respectivamente. Como $F$ é bi-Lipschitz com constantes $0<c_{1} \leqslant c_{2}$,

$$
\|p\| \geqslant r \Rightarrow\|F(p)\| \geqslant c_{1} r
$$

e, portanto,

$$
\begin{aligned}
r^{m} h(r) & =\left\|x_{2}(r)-x_{j}(r)\right\| \\
& \geqslant \frac{1}{c_{2}}\left\|F\left(x_{i}(r)\right)-F\left(x_{j}(r)\right)\right\| \\
& =\frac{1}{c_{2}}\left(c_{1} r\right)^{n} g\left(c_{1} r\right) .
\end{aligned}
$$


Com isso, mostramos que $m \leqslant n$. Simetricamente, podemos mostrar que $n \leqslant m$ e, portanto, $m=n$.

Reciprocamente, suponhamos $I=J$ e $\operatorname{sh}\left(X_{i}, X_{j}\right)=\operatorname{sh}\left(Y_{i}, Y_{j}\right)$ para cada $i \neq j$. Definamos $F:(X, 0) \rightarrow(Y, 0)$ por: dado $x \in X_{i} \cup S_{r}(0)$, seja $F(x)$ o ponto na interseçāo $Y_{i} \cap S_{r}(0)$. Segue imediatamente da. Proposição 2.1.2 que $F$ é o germe de uma aplicação bi-Lipschitz.

\subsection{Sobre o tipo Lipschitz de curvas reais}

Sejam $X, Y \subset \mathbb{R}^{n}$, subconjuntos semi-algébricos de dimensão real 1, e sejam $x \in X$, $y \in Y$.

Nesta seção, procuramos responder à seguinte pergunta: quando existe um germe de aplicação semi-algébrica bi-Lipschitz $F:\left(\mathbb{R}^{n}, x\right) \rightarrow\left(\mathbb{R}^{n}, y\right)$ tal que $F(X)=Y$ ?

Sejam $(X, x),(Y, y) \subset \mathbb{R}^{n}$ germes de curvas semi-algébricas, com semi-ramos $X=\bigcup_{i \in I} X_{i}$ e $Y=\bigcup_{i \in J} X_{j}$. Para efeito de simplicidade, nas demonstraçōes e em alguns resultados auxiliares deste capítulo, escrevemos $\operatorname{sh}(X, x)=\operatorname{sh}(Y, y)$ para indicar que existe uma bijeção $\phi: I \rightarrow J$ tal que $\operatorname{sh}\left(X_{i}, X_{j}\right)=\operatorname{sh}\left(Y_{\phi(i)}, Y_{\phi(j)}\right)$, para cada par $i \neq j \in I$.

Segue do Teorema 2.1.3 que $\operatorname{sh}(X, x)=\operatorname{sh}(Y, y)$ é uma condição necessária para que exista um germe de aplicação semi-algébrica bi-Lipschitz $F:\left(\mathbb{R}^{n}, x\right) \rightarrow\left(\mathbb{R}^{n}, y\right)$ tal que $F(X)=Y$. Nesta seção, mostramos que tal condição, é também suficiente.

Respondemos à pergunta formulada no início desta seção em duas etapas. Na primeira etapa, a qual tem início a seguir, respondemos essa pergunta para o caso de curvas planas e, na segunda etapa, concluímos o resultado para o caso geral.

Denotamos o primeiro quadrante de $\mathbb{R}^{2}$ por $Q=\left\{(x, y) \in \mathbb{R}^{2}: x \geq 0\right.$ e $\left.y \geq 0\right\}$ e dada uma função $f:[0, \delta] \rightarrow \mathbb{R}$, denotamos $T_{f}=\left\{(x, y) \in \mathbb{R}^{2}: x \geq 0\right.$ e $\left.y=f(x)\right\}$. Quando $f(x)=x^{\alpha}$, denotamos $T_{\alpha}=T_{f}$.

Lema 2.2.1. Sejam $f:[0, \delta] \rightarrow \mathbb{R}$ uma função semi-algébrica com decomposiçâao de Newton-Puiseux em $x=0$, dada por $f(x)=x^{\alpha} h(x) \operatorname{com} h(0)>0$. Consideremos $o$ 
seguinte germe de aplicação semi-algébrica $F:\left(\mathbb{R}^{2}, 0\right) \rightarrow\left(\mathbb{R}^{2}, 0\right)$; para cada $(x, y) \in$ $Q$, é definido por:

$$
F(x, y)=\left\{\begin{array}{lll}
\left(x, \frac{y}{h(x)}\right) & \text { se } 0 \leq y \leq f(x) \\
\left(x, y-f(x)+x^{\alpha}\right) & \text { se } 0 \leq f(x) \leq y
\end{array}\right.
$$

e, fora de $Q, F$ é definido como o germe da aplicação identidade. Então, $F$ é um germe de aplicação semi-algébrica bi-Lipschitz tal que $\left(F\left(T_{f}\right), 0\right)=\left(T_{\alpha}, 0\right)$ e fora do quadrante $Q$ coincide com a aplicação identidade.

Prova. É claro que $F$ define um germe de homeomorfismo semi-algébrico tal que $\left(F\left(T_{f}\right), 0\right)=\left(T_{\alpha}, 0\right)$. Também é claro que $F$ é bi-Lipschitz fora do domínio: $\{(x, y) \in$ $Q ; 0 \leqslant y \leqslant f(x)\}$. Mas, nesse domínio, a Proposição 4.1 de [2] mostra que $F$ é bi-Lipschitz.

O Lema 2.2.1 é fundamental para a solução que apresentamos para o problema de classificação do tipo semi-algébrico bi-Lipschitz de curvas reais. Os resultados que apresentamos nesta seção, foram obtidos quando visitei a Universidade de Valladolid em Abril-Maio de 2001 e formam parte de um artigo, ainda em preparação, junto com o Professor Dr. Lev Birbrair, sobre classificação bi-Lipschitz de singularidades (o-minimais) de dimensão real 1 e 2.

Observação 2.2.2. Como conseqüência do Lema 2.2.1, temos que se $f, g:[0, \delta] \rightarrow \mathbb{R}$ são funções semi-algébricas, com os mesmos primeiros expoentes de Newton-Puiseux em $x=0$, então existe um germe de aplicação semi-algébrica bi-Lipschitz $F$ : $\left(\mathbb{R}^{2}, 0\right) \rightarrow\left(\mathbb{R}^{2}, 0\right)$ tal que $F\left(T_{f}\right)=T_{g}$.

Lema 2.2.3. Sejam $f_{i}, g_{i}:[0, \delta] \rightarrow \mathbb{R}$ funções semi-algébricas, com $f_{i}(0)=g_{i}(0)=0$ e $i=1, \ldots, m$. Sejam também, $X=\bigcup \operatorname{Graf}\left(f_{i}\right)$ e $Y=\bigcup \operatorname{Graf}\left(g_{i}\right)$. Se $\operatorname{sh}(X, 0)=$ $\operatorname{sh}(Y, 0)$, então existe um germe de aplicação semi-algébrica bi-Lipschitz $F:\left(\mathbb{R}^{2}, 0\right) \rightarrow$ $\left(\mathbb{R}^{2}, 0\right)$ tal $\cdot q u e ~ F(X)=Y$ e, fora do quadrante $Q, F$ coincide com a aplicaçäo identidade. 
Prova. Provamos o resultado acima por indução 'sobre $m \geq 1$. Antes, podemos fazer algumas considerações sem perda de generalidade. Por exemplo, podemos supor $0 \equiv$ $f_{m} \leq \ldots \leq f_{1}$ e $0 \equiv g_{m} \leq \ldots \leq g_{1}$. O caso em que $m=2$, segue imediatamente do Lema 2.2.1. Agora, suponhamos $m>2$ e, por induçäo, suponhamos que o resultado seja válido para $m-1$. Novamente, pelo Lema 2.2.1, podemos supor $f_{m-1}=h=g_{m-1}$. Então, sejam $\tilde{X}=\bigcup_{i=1}^{m-1} \operatorname{Graf}\left(\tilde{f}_{i}\right), \tilde{Y}=\bigcup_{i=1}^{m-1} \operatorname{Graf}\left(\tilde{g}_{i}\right), \tilde{f}_{\mathfrak{i}}=f_{i}-h$ e $\tilde{g}_{i}=g_{i}-h$, $i=1, \ldots, m-1$. Por hipótese de indução, existe um germe de aplicação semialgébrica bi-Lipschitz $\widetilde{F}:\left(\mathbb{R}^{2}, 0\right) \rightarrow\left(\mathbb{R}^{2}, 0\right)$ tal que $\widetilde{F}(\widetilde{X})=\widetilde{Y}$ e, fora de $Q, \widetilde{F}$ coincide com a aplicaçāo identidade.

Tómemos, $\Phi:\left(\mathbb{R}^{2}, 0\right) \rightarrow\left(\mathbb{R}^{2}, 0\right)$ dada por $\Phi(x, y)=(x, y-h(x))$. Assim, $F:$ $\left(\mathbb{R}^{2}, 0\right) \rightarrow\left(\mathbb{R}^{2}, 0\right)$ dada por $F=\Phi^{-1} \circ \widetilde{F} \circ \Phi$ é um germe de aplicação semi-algébrica bi-Lipschitz tal que $(F(X), 0)=(Y, 0)$ e $F$ coincide com a aplicação identidade fora de $Q$.

Observação 2.2.4. Sejam $c>0$ e $K=\left\{(x, y) \in \mathbb{R}^{2}: 0 \leq x\right.$ e $\left.0 \leq y \leq c x\right\}$. Então, $\Phi: \mathbb{R}^{2}, 0 \rightarrow \mathbb{R}^{2}, 0$, dada por $\Phi(x, y)=(c x-y, y)$, é uma aplicação semialgébrica bi-Lipschitz tal que $\Phi(K)=Q$. Com isso, podemos reenunciar o Lema 2.2.3 da seguinte forma:

Lema 2.2.5. Sejam $f_{i}, g_{i}:[0, \delta] \rightarrow \mathbb{R}$ funçōes semi-algébricas, com $f_{i}(0)=g_{i}(0)=0$ e $i=1, \ldots, m$. Sejam também, $X=\bigcup \operatorname{Graf}\left(f_{i}\right), Y=\bigcup \operatorname{Graf}\left(g_{i}\right), c>0$ e $K=$ $\left\{(x, y) \in \mathbb{R}^{2}: 0 \leq x e 0 \leq y \leq c x\right\}$ cone contendo $X$ e $Y \cdot S e \operatorname{sh}(X, 0)=\operatorname{sh}(Y, 0)$, então existe um germe de aplicação semi-algébrica bi-Lipschitz $F:\left(\mathbb{R}^{2}, 0\right) \rightarrow\left(\mathbb{R}^{2}, 0\right)$ tal que $F(X)=Y$ e, fora do cone $K, F$ coincide com a aplicação identidade.

Lema 2.2.6. Sejam $X, Y \subset \mathbb{R}^{2}$ curvas semi-algébricas e sejam $x \in X, y \in Y$. Se $\operatorname{sh}(X, x)=\operatorname{sh}(Y, y)$, então existe um germe de aplicação semi-algébrica bi-Lipschitz $F:\left(\mathbb{R}^{2}, 0\right) \rightarrow\left(\mathbb{R}^{2}, 0\right)$ tal que $F(X)=Y$.

Prova. Sem perda de generalidade, podemos supor $x=0=y \in \mathbb{R}^{2}$. Por hipótese, $\operatorname{sh}(X, 0)=\operatorname{sh}(Y, 0)$. Portanto, o número $t$ de semi-retas reais tangentes a $(X, 0)$ coincide com o número de semi-retas reais tangentes a $(Y, 0)$. Separemos os semi-ramos de 
$(X, 0)$, respectivamente $(Y, 0)$, em cones $K_{1}^{X}, \ldots, K_{t}^{X}$, respectivamente $K_{1}^{Y}, \ldots, K_{t}^{Y}$, os quais possuem interseção somente na origem, de tal maneira que dois semi-ramos de $(X, 0)$, respectivamente de $(Y, 0)$, estāo em um mesmo cone se, e somente se, possuem o mesmo vetor unitário tangente na origem. Como $\operatorname{sh}(X, 0)=\operatorname{sh}(Y, 0)$, podemos enumerar os cones de forma que:

$$
\operatorname{sh}\left(X \cap K_{i}^{X}, 0\right)=\operatorname{sh}\left(Y \cap K_{i}^{Y}, 0\right)
$$

para cada $i=1, \ldots, t$. Agora, pelo Lema 2.2.5, para cada $i=1, \ldots, t$, temos um germe de aplicação semi-algébrica bi-Lipschitz

$$
F_{i}:\left(\mathbb{R}^{2}, 0\right) \rightarrow\left(\mathbb{R}^{2}, 0\right)
$$

tal que $\left.\left(F_{i}\left(X \cap K_{i}^{X}\right)\right)=Y \cap K_{i}^{Y}\right)$ e $F_{i}$ coincide com a aplicação identidade fora do cone $K_{i}^{X}$. Então, seja

$$
F:\left(\mathbb{R}^{2}, 0\right) \rightarrow\left(\mathbb{R}^{2}, 0\right)
$$

dada por: $F(x, y)=F_{i}(x, y)$ caso exista $i$ tal que $(x, y) \in K_{i}^{X}$ e $F(x, y)=(x, y)$ no caso contrário. Temos que $F$ é um germe de aplicação semi-algébrica bi-Lipschitz tal que $(F(X), 0)=(Y, 0)$.

Teorema 2.2.7. Sejam $(X, x),(Y, y) \subset \mathbb{R}^{n}$ germes de curvas semi-algébricas, com semi-ramos $X=\bigcup_{i \in I} X_{i}$ e $Y .=\bigcup_{i \in J} X_{j}$. Se existe uma bijeçâo $\phi: I \rightarrow J$ tal que tal que $\operatorname{sh}\left(X_{i}, X_{j}\right)=\operatorname{sh}\left(Y_{\phi(i)}, Y_{\phi(j)}\right)$, para cada par $i \neq j \in I$, então existe um germe de aplicação semi-algébrica bi-Lipschitz $F:\left(\mathbb{R}^{n}, x\right) \rightarrow\left(\mathbb{R}^{n}, y\right)$ tal que $F(X)=Y$.

Prova. Provaremos este teorema por indução sobre o número $n \geqslant 2$. O Caso $n=2$ segue imediatamente do Lema 2.2.6. Então, sejam $n \geqslant 2$ e $(X, x),(Y, y)$ germes de curvas semi-algébricas em $\mathbb{R}^{n+1}$ tais que $\operatorname{sh}(X, x)=\operatorname{sh}(Y, y)$. Suponhamos, por hipótese de indução, que o teorema seja verdadeiro para germes de curvas semialgébricas em $\mathbb{R}^{n}$. Sem perda de generalidade, podemos supor $x=y=0 \in \mathbb{R}^{n+1}$.

Sejam $\Pi_{1}, \Pi_{2}: \mathbb{R}^{n+1}, 0 \rightarrow \mathbb{R}^{n}, 0$ projeçōes genéricas tais que

$$
\operatorname{sh}\left(\Pi_{1}(X), 0\right)=\operatorname{sh}(X, 0) \quad \text { e } \operatorname{sh}\left(\Pi_{2}(Y), 0\right)=\operatorname{sh}(Y, 0) .
$$


Entāo, temos germes de funções semi-algébricas bi-Lipschitz

$$
f_{1}:\left(\Pi_{1}(X), 0\right) \rightarrow(\mathbb{R}, 0) \quad f_{2}:\left(\Pi_{2}(Y), 0\right) \rightarrow(\mathbb{R}, 0)
$$

tais que $\operatorname{Graf}\left(f_{1}\right)=X$ e $\operatorname{Graf}\left(f_{2}\right)=Y$. Pelo Teorema de Kirszbraun, existem extensões semi-algébricas bi-Lipschitz $\phi_{1}, \phi_{2}:\left(\mathbb{R}^{n}, 0\right) \rightarrow(\mathbb{R}, 0)$ de $f_{1}, f_{2}$ respectivamente. Agora, consideremos os seguintes germes de aplicações semi-algébricas biLipschitz $F_{1}, F_{2}:\left(\mathbb{R}^{n+1}, 0\right) \rightarrow\left(\mathbb{R}^{n+1}, 0\right)$ dados por

$$
F_{i}\left(z_{1}, \ldots, z_{n}, z_{n+1}\right)=\left(z_{1}, \ldots, z_{n}, z_{n+1}-\phi_{i}\left(z_{1}, \ldots, z_{n}\right)\right), i=1,2 \text {. }
$$

Temos $\tilde{X}, \widetilde{Y}$ curvas semi-algébricas em $\mathbb{R}^{n}$ tais que

$$
\left(F_{1}(X), 0\right)=(\widetilde{X} \times 0,0) \quad\left(F_{2}(Y), 0\right)=(\widetilde{Y} \times 0,0)
$$

Daí,

$$
\operatorname{sh}(\widetilde{X}, 0)=\operatorname{sh}(X, 0)=\operatorname{sh}(Y, 0)=\operatorname{sh}(\widetilde{Y}, 0)
$$

Logo, por hipótese de induçāo, existe um germe de aplicação semi-algébrica biLipschitz $\widetilde{F}:\left(\mathbb{R}^{n}, 0\right) \rightarrow\left(\mathbb{R}^{n}, 0\right)$ tal que $\widetilde{F}(\widetilde{X})=\widetilde{Y}$. Finalmente, sejam $F, F_{3}$ : $\left(\mathbb{R}^{n+1}, 0\right) \rightarrow\left(\mathbb{R}^{n+1}, 0\right)$ dadas por

$$
F_{3}\left(z_{1}, \ldots, z_{n}, z_{n+1}\right)=\left(\widetilde{F}\left(z_{1}, \ldots, z_{n}\right), z_{n+1}\right)
$$

e $F=F_{2}^{-1} \circ F_{3} \circ F_{1}$. Assim, $F$ é um germe de aplicação semi-algébrica bi-Lipschitz tal que $F(X)=Y$.

\subsection{Sobre famílias de curvas reais}

Definição 2.3.1. Seja $Y$ um subconjunto semi-algébrico de $\mathbb{R}^{p}$. Uma família de germes de conjuntos semi-algébricos em $\mathbb{R}^{n} \times \mathbb{R}^{p}$ sobre $Y$ é um germe $X$ em $\{0\}^{n} \times Y$ de subconjunto de $\mathbb{R}^{n} \times \mathbb{R}^{p}$ tal que o germe de $X$ em cada ponto $(0, t)$ é semi-algébrico. Denotamos II : $\mathbb{R}^{n} \times Y \rightarrow Y$ projeção canônica e $X_{t}=X \cap \Pi^{-1}(t)$. 
Dizemos que $X$ é topologicamente (respect. bi-Lipschitz) trivial ao longo de $Y$ se. existe um germe de homeomorfismo (respect. homeomorfismo bi-Lipschitz)

$$
H:\left(\mathbb{R}^{n} \times Y,\{0\}^{n} \times Y\right) \rightarrow\left(\mathbb{R}^{n} \times Y,\{0\}^{n} \times Y\right)
$$

tal que $\Pi \circ H=\Pi$ e $H(X)=X_{t_{0}} \times Y$ para algum $t_{0} \in Y$

Observação 2.3.2. Seja $X$ uma família de germes de curvas semi-algébricas pela origem de $\mathbb{R}^{n}$, ao longo de um intervalo $I$. Dizer que $\operatorname{sh}\left(X_{t}, 0\right)$ é constante significa dizer que existem $\delta>0$ e $m \geq 1$ tais que para cada $t \in I$ temos uma quantidade $m$ de semi-ramos de $\left(X_{t}, 0\right)$

$$
X_{t}^{1}, \ldots, X_{t}^{m}
$$

e existem $0<K_{1} \leq K_{2}$ e uma coleção $\alpha_{i, j}$ de números racionais tais que

$$
x_{i}(r, t)-x_{j}(r, t)=r^{\alpha_{i, j}} d_{i, j}(r, t)
$$

em que $K_{1} \leq\left\|d_{i, j}(r, t)\right\| \leq K_{2}, \quad \forall(r, t) \in[0, \delta] \times I$ e $x_{i}(r, t)$ é o ponto na interseção de $X_{t}^{i}$ com a esfera de raio $r$ e centro na origem.

De acordo com as considerações acima, temos claramente que para que uma família de curvas $X_{t}$ pela origem de $\mathbb{R}^{n}$ seja bi-Lipschitz trivial é necessário que $\operatorname{sh}\left(X_{t}, 0\right)$ seja constante.

Exemplo 2.3.3. (Kite singularities [19])

Sejam $1<k<l<m$ e consideremos

$$
X_{t}=\left\{(x, y): y^{2 k}=x^{2 m+1}+t x^{2 l+1}\right\}
$$

Temos que: $\operatorname{sh}\left(X_{t}, 0\right)=\frac{2 l+1}{2 k}$ para $t \neq 0$ e $\operatorname{sh}\left(X_{0}, 0\right)=\frac{2 m+1}{2 k}$. Portanto, para qualquer intervalo não degenerado $I$ de $\mathbb{R}$ contento 0 , a família $X_{t}$ não é bi-Lipschitz trivial ao longo desse intervalo. Por outro lado, é bem conhecido que essa família é Whitney equisingular.

O exemplo acima deixa clara a diferença entre estratificaçōes de Whitney e estratificações bi-Lipschitz. Pois, estratificações de Whitney oferecem o bom comportamento de pares de estratos de dimensões distintas enquanto para que uma estratificação 
seja bi-Lipschitz trivial, também devemos ter o bom comportamento de pares de estratos de mesma dimensão. E, para o caso de família de curvas, esse bom comportamento de pares de estratos de mesma dimensão vai ser obtido a partir de hipóteses sobre $\operatorname{sh}\left(X_{t}, 0\right)$.

Proposição 2.3.4. A familia (2.1) de quatro retas de H. Whitney é bi-Lipschitz trivial.

Prova. Consideremos $X=\{(x, y, z): x y(x-y)(x-z y)=0, z>1\}$. Para cada $t>1$, seja $X_{t}=\{(x, y, z) \in X: z=t$. Consideremos os seguintes subconjuntos de $X:$

- $X^{0}=\{(x, y, z) \in X: x=0\}$;

- $X^{1}=\{(x, y, z) \in X: y=0\}$;

- $X^{2}=\{(x, y, z) \in X: x=y\}$

- $X^{3}=\{(x, y, z) \in X \quad: x=z y\}$.

Definamos $V: X \rightarrow \mathbb{R}^{3}$, campo de vetores em $X$, da seguinte forma:

$$
V(x, y, z)=\left\{\begin{array}{lll}
(0,0,1) & \text { se } & (x, y, z) \notin X^{3} \\
(y, 0,1) & \text { se } & (x, y, z) \in X^{3}
\end{array}\right.
$$

É claro que $V$ é um campo tangente a cada $X^{i} ; i=0,1,2,3$. Nesse momento, consideramos $\mathbb{R}^{3}$ equipado da métrica da soma.

Afirmação. Para cada $t>1, V_{t}:=V(\cdot, t)$ é um campo $k(t)$-Lipschitz, com $k(t)$ contínua em $t$.

Com efeito, sejam $P=(x, y, t)$ e $Q=(a, b, t)$ pontos de $X$. Se $P, Q \notin X^{3}$, então $V(P)=V(Q)$. Assim, podemos supor que $P \in X^{3}$, isto $\dot{e}, P=(t y, y, t)$.

- Se $Q \in X^{0}$, então $\|V(P)-V(Q)\| \leqslant t^{-1}\|P-Q\|$

- Se $Q \in X^{1}$, então $\|V(P)-V(Q)\| \leqslant\|P-Q\|$

- Se $Q \in X^{2}$, então $\|V(P)-V(Q)\| \leqslant(t-1)^{-1}\|P-Q\|$; 
- $Q \in X^{0}$, entāo $\|V(P)-V(Q)\| \leqslant(t+1)^{-1}\|P-Q\|$.

Assim, temos $V_{t}$ um campo $k(t)$-Lipschitz para $k(t)=\max \left\{1,(1-t)^{-1}\right\}$. Fica provada a afirmação acima.

Seja $I$ um intervalo compacto em $(1,+\infty)$. Pela afirmação acima, $V_{t}$ é um campo $k$-Lipschitz com a constante $k$ independendo de $t$. Usando o Teorema de Kirzbraun, temos uma extensão $W: \mathbb{R}^{2} \times I \rightarrow \mathbb{R}^{3}$ de $V$ tal que para cada $t \in I, W_{t}:=W(\cdot, t)$ é um campo $k$-Lipschitz. Agora, como $V$ é tangente a $X$, temos que o fluxo de $W$ nos dá uma trivializaçāo bi-Lipschitz de $X$ ao longo de $I$.

Teorema 2.3.5. Seja $X$ uma família de germes de curvas semi-algébricas pela origem de $\mathbb{R}^{n}$, ao longo de um intervalo $I$. Se $X$ é Whitney equisingular ao longo de $I e$ $\operatorname{sh}\left(X_{t}, 0\right)$ é constante, então $X$ é bi-Lipschitz trivial ao longo de $I$.

Prova. Como $X$ é Whitney equisingular ao longo de $I=[0, a]$, podemos considerar $V$ um campo de vetores, integrável, sobre $X ; V$ coincide com $\frac{\partial}{\partial t}$ sobre o $t$-eixo e seu fluxo $\phi$ sobre $X$ satifaz: $\left\|\phi_{t}(x)\right\|=\|x\|, \forall(x, t)$ (cf. [16]). Usando a notação da observação acima, isto significa que $x_{i}(r, t)=x_{i}(r, 0), \forall i$. Então ,

$$
\begin{aligned}
\left\|V_{t}\left(x_{i}(r, t)\right)-V_{t}\left(x_{j}(r, t)\right)\right\| & =r^{\alpha_{i, j}}\left\|\frac{\partial}{\partial t} d_{i, j}(r, t)\right\| \\
& \leq K\left\|x_{i}(r, t)-x_{j}(r, t)\right\| .
\end{aligned}
$$

Assim, pelo Lema 2.1.2, segue que, para cada $t \in I, V_{t}$ é um campo Lipschitz com uma constante que não depende de $t$. Com isso, podemos usar o Teorema de Kirszbraun para estender $V$ a um campo de vetores Lipschitz sobre $\mathbb{R}^{n} \times I$ e tomar o seu fluxo para obter uma trivialização bi-Lipschitz de $X$.

Observaçāo 2.3.6. No Teorema 2.3 .5 é suficiente pedirmos que a família $X_{t}$ seja c-regular no sentido de K. Bekka [1], uma vez que $X_{t}$ c-regular ao longo de $I$ ainda é suficiente para garantir a existência de $\phi_{t}$ satisfał́zendo $\left\|\phi_{t}(x)\right\|=\|x\|, \forall(x, t)$.

Lev Birbrair, em conversa particular, apontou-me para a dificuldade de se estabelecer teoremas de existência e caracterização de estratificaçōes semi-algebricamente 
bi-Lipschitz triviais. Pois, a integração de campos semi-algébricos não fornece aplicaçōes semi-algébricas. A seguir, apresentamos um resultado de caracterização de famílias de curvas planas semi-algebricamente bi-Lipschitz triviais.

Definiçāo 2.3.7. Seja $Y$ um subconjunto semi-algébrico de $\mathbb{R}^{p}$. Uma familia de germes de conjuntos semi-algébricos em $\mathbb{R}^{n} \times \mathbb{R}^{p}$ sobre $Y$ é um germe $X$ em $\{0\}^{n} \times Y$ de subconjunto de $\mathbb{R}^{n} \times \mathbb{R}^{p}$ tal que o germe de $X$ em cada ponto $(0, t)$ é semi-algébrico. Denotamos por $\Pi: \mathbb{R}^{n} \times Y \rightarrow Y$ a projeção canônica e $X_{t}=X \cap \Pi^{-1}(t)$.

Dizemos que $X$ é semi-algebricamente bi-Lipschitz trivial ao longo de $Y$ se existe um germe de homeomorfismo semi-algébrico bi-Lipschitz

$$
H:\left(\mathbb{R}^{n} \times Y,\{0\}^{n} \times Y\right) \rightarrow\left(\mathbb{R}^{n} \times Y,\{0\}^{n} \times Y\right)
$$

tal que $\Pi \circ H=\Pi$ e $H(X)=X_{t_{0}} \times Y$ para algum $t_{0} \in Y$.

Observação 2.3.8. Seja $X$ uma família semi-algébrica de germes de curvas semialgébricas planas, pela origem de $\mathbb{R}^{2}$, ao longo de um intervalo compacto $I$ de $\mathbb{R}$. É um fato que, para cada $t \in I$ existe uma vizinhança da origem $U_{t}$ em $\mathbb{R}^{2}$ tal que $X_{t} \cap U_{t}$ tem os seus semi-ramos, na origem, dados por gráficos de funções semialgébricas convexas. Em particular, estas funções, quando não são identicamente nulas, não se anulam em pontos desse domínio. Nesse caso, diremos que $X_{t} \cap U_{t}$ tem uma estrutura de gráfico (na origem).

Definiçāo 2.3.9. Seja $X$ uma família semi-algébrica de germes de curvas semialgébricas planas, pela origem de $\mathbb{R}^{2}$, ao longo de um intervalo compacto $I$ de $\mathbb{R}$. Dizemos que $X$ tem estrutura uniforme de gráfico (na origem), se existe uma vizinhança $U$ da origem em $\mathbb{R}^{2}$ tal que para cada $t \in I, U \cap X_{t}$ tem uma estrutura de gráfico (na origem).

Dado um germe de curva semi-algébrica $(X, 0)$, seu cone tangente, que denotamos por $c(X)$, é o conjunto formado pelas semi-retas tangentes a $(X, 0)$.

Lema 2.3.10. Seja $X$ uma familia semi-algébrica de germes de curvas semi-algébricas pela origem de $\mathbb{R}^{2}$, ao longo de um intervalo $I=[a, b]$ de $\mathbb{R}$, tal que a familia de $c\left(X_{t}\right)$ 
associada a $X$ é trivial. Se $X$ é topologicamente trivial, ao longo de $I$, então $X$ possui uma estrutura uniforme de gráfico (na origem).

Prova. Seja $B$ uma bola em torno da origem de $\mathbb{R}^{2}$ tal que $X_{a} \cap B$ possua estrutura de gráfico (na origem). Como $X$ é topologicamente trivial, ao longo de $I$, podemos supor que $B$ é suficientemente pequena tal que para cada aberto $V \subset B$, em torno da origem, temos: para cada $t, s \in I$, existe um homeomorfismo $\phi_{t, s}: V \rightarrow \dot{V}$; $\phi_{t, s}\left(X_{t} \cap V\right)=\left(X_{s} \cap V\right)$ e $\phi_{t, s}(0)=0$.

Tomemos $[0, \delta]$ o maior intervalo contido na projeção ortogonal de $X_{t} \cap B$ sobre a sua semi-reta real tangente $l, \forall t$. Observemos que $0<\delta$, pois caso contrário, usando a compacidade de $I$, mostraríamos que, para algum $t, X_{t} \cap B$ intersectaria a reta. ortogonal a $l$ e isto contradiz o fato de termos um homeormofismo $\phi: B \rightarrow B$ tal que $\phi\left(X_{a} \cap B\right)=X_{t} \cap B$. Agora tomemos $V=B \cap[-\delta, \delta] \times \mathbb{R}$. Então, dado $t$, qualquer reta vertical contida em $V$ intersecta $X_{t}$

Por contradição, suponhamos que uma curva $X_{t} \cap B$ possua um semi-ramo, na origem, que não seja gráfico sobre sua tangente $l$. Isto é, temos uma reta $r_{1}$ vertical (reta ortogonal a $l$ ) tal que, se $r_{1}$ intersecta esse semi-ramo o faz mais de uma vez. Então, tomemos um aberto $V \subset B$ em torno da origem tal que $r_{1} \cap V \subset \partial V$. Observe que isso contradiz o fato de existir um homeomorfismo $\phi_{a, t}: V \rightarrow V ; \phi_{a, t}\left(X_{a} \cap V\right)=$ $\left(X_{t} \cap V\right)$ e $\phi_{a, t}(0)=0$ pois, como $X_{a} \cap V$ possui uma estrutura de gráfico (na origem), $r_{1} \cap B$ intersecta cada semi-ramo de $X_{a} \cap V$ (na origem) uma, e somente uma, vez.

Também, por contradição. e tomando retas horizontais (retas paralelas a uma determinada tangente $l$ ), podemos provar que para cada $t \in I$ os semi-ramos da curva $X_{t}$ (na origem), com tangente $l$, ou bem coincidem com $l$ ou não intersectam $l$.

Definição 2.3.11. Uma família semi-algébrica $X$ de germes de curvas semi-algébricas pela origem de $\mathbb{R}^{n}$ topologicamente trivial ao longo de um intervalo $I=[a, b]$ de $\mathbb{R}$ é ct-regular se a familia de cones tangentes $c\left(X_{t}\right)$ é semi-algebricamente topologicamente trivial.

Exemplo 2.3.12. (Teissier, 1974) 
A seguinte família de curvas planas pela origem de $\mathbb{R}^{2}$

$$
X_{t}: y^{3}=x^{5}+t x^{2}
$$

nāo possui uma estrutura uniforme de gráfico (na origem), embora possua tipo topológico constante. Vale observar que, para cada $t, X_{t}$ possui dois semi-ramos reais pela origem com contato igual a 1 .

Exemplo 2.3.13. A seguinte família de germes de curvas planas

$$
\cos \left(t+\frac{\pi}{2}\right) x+\sin \left(t+\frac{\pi}{2}\right)\left(x^{2} y+y^{3}\right)=0
$$

sugerida por R.N.A. dos Santos [26], é topologicamente trivial, mas não é ct-regular.

Notação. Uma função $f: I \times[0, \delta] \rightarrow \mathbb{R}$ define uma família de funçōes $f_{t}$ : $[0, \delta] \rightarrow \mathbb{R} ; \forall t \in I, f_{t}(x)=f(x, t)$. E, denotamos

$$
T_{f}=\left\{(x, y, t) \in \mathbb{R}^{3} \mid(x, y) \in T_{f_{t}}\right\}
$$

Lema 2.3.14. Seja $f: I \times[0, \delta] \rightarrow \mathbb{R}$ uma função semi-algébrica tal que, para cada $t \in I, f_{t}$ é uma função não negativa com decomposição de Newton-Puiseux em $x=0$ dada por $f_{t}(x)=x^{\alpha} h_{t}(x)$. Então,

$$
\left.F:\left(\mathbb{R}^{2} \times \mathbb{R}, 0\right) \rightarrow \mathbb{R}^{2} \times \mathbb{R}, 0\right)
$$

dada por $F(x, y, t)=\left(F_{t}(x, y), t\right)$ em que

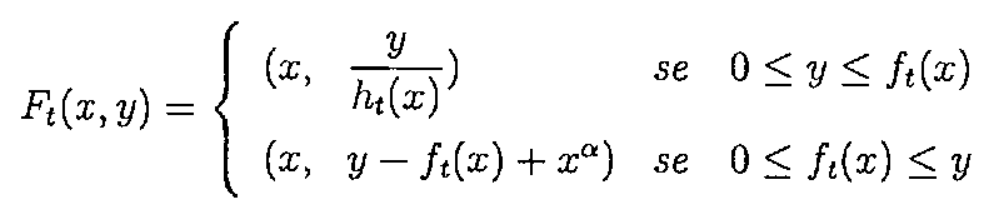

é um germe de aplicação semi-algébrica bi-Lipschitz tal que $\Pi \circ F=\Pi$ e $F\left(T_{f}\right)=$ $T_{\alpha} \times \mathbb{R}$. Além disso, para cada $t \in I, F_{t}$ coincide com a aplicação identidade fora do quadrante $Q$.

Como aplicação do Lema 2.3.14, vejamos o seguinte exemplo. Consideremos a seguinte família de germes de curvas semi-algébricas planas, pela origem de $\mathbb{R}^{2}$ :

$$
X_{t}: x^{6}-y^{4}+t x^{3} y^{2}=0
$$


Mostremos que essa família é semi-algebricamente bi-Lipschitz trivial ao longo de qualquer intervalo $I=[-a, a], a>0$. Por simetria, podemos considerar

$$
X_{t}: x^{6}-y^{4}+t x^{3} y^{2}=0 ; x \geq 0
$$

Temos $X_{t} \cap\{x=1\}=\{(1, g(t)),(1,-g(t))\}$, em que $g$ é uma função semialgébrica, contínua, tal que $g(0)>0$. Como $X_{t}$ é homogêneo com relação aos pesos $w t(x)=2$ e $w t(y)=3$, temos que os semi-ramos de $X_{t}$ na origem são parametrizados por

$$
\left(s^{2}, s^{3} g(t)\right) \quad: \quad\left(s^{2},-s^{3} g(t)\right) ; s \geq 0
$$

Ou ainda, temos que os semi-ramos de $X_{t}$ na origem são dados pelos gráficos

$$
y=x^{\frac{3}{2}} g(t) \quad: \quad y=-x^{\frac{3}{2}} g(t) \quad ; x \geq 0
$$

Após considerarmos a seguinte mudança de coordenadas bi-Lipschitz

$$
(x, y, t) \mapsto\left(x, y+x^{\frac{3}{2}} g(t), t\right)
$$

temos que $X_{t}$ pode ser visto como os gráficos

$$
y=0 \quad: \quad y=2 x^{\frac{3}{2}} g(t) \quad ; x \geq 0
$$

isto é, $X_{t}=T_{f_{t}}$ em que $f_{t}(x)=2 x^{\frac{3}{2}} g(t)$. Segue do Lema 2.3.14, que a família $X=\left\{(x, y, t):(x, y) \in X_{t}\right\}$ é semi-algebricamente bi-Lipschitz trivial ao longo de $I$.

Teorema 2.3.15. Sejam $X$ e $Y$ duas familias de germes de curvas semi-algébricas pela origem de $\mathbb{R}^{2}$, ct-regulares ao longo de um intervalo $I$ e topologicamente equivalentes. Se $\operatorname{sh}\left(X_{t}, 0\right)=\operatorname{sh}\left(Y_{t}, 0\right), \forall t \in I$, então $X$ e $Y$ são semi-algebricamente biLipschitz equivalentes. Em particular, se $X$ é ct-regular, topologicamente trivial $e$ $\operatorname{sh}\left(X_{t}, 0\right)$ é constante, então $X$ é semi-algebricamente bi-Lipschitz trivial.

Prova. Idêntica à prova dada para o Lema 2.2.6, aplicando aqui a versão do Lema 2.2 .5 para famílias. 


\subsection{Sobre famílias de curvas reais quase-homogêneas}

Seja $\left(a_{1}, \ldots, a_{n}\right)$ uma $n$-upla de inteiros positivos primos entre si. Associada a $\left(a_{1}, \ldots, a_{n}\right)$ temos a seguinte ação :

$$
t \cdot p:=\left(t^{a_{1}} p_{1}, \ldots, t^{a_{n}} p_{n}\right) \quad \text { para cada } t \in \mathbb{R}^{*} \quad \text { e } \quad p=\left(p_{1}, \ldots, p_{n}\right) \in \mathbb{R}^{n}
$$

Definição 2.4.1. Uma aplicação $F: \mathbb{R}^{n} \rightarrow \mathbb{R}^{m} ; F=\left(f_{1}, \ldots, f_{m}\right)$ é quase-homogênea de tipo $\left(a_{1}, \ldots, a_{n}: d_{1}, \ldots, d_{m}\right)$ se $F(t \cdot p)=\left(t^{d_{1}} f_{1}(p), \ldots, t^{d_{m}} f_{m}(p)\right)$ para cada $t \in \mathbb{R}$ e $p=\left(p_{1}, \ldots, p_{n}\right) \in \mathbb{R}^{n}$.

O exemplo das quatro retas de H.Whitney (2.1) é um exemplo de uma família de conjuntos singulares definidos como zeros de uma família de aplicações homogêneas que apresenta modalidade para o problema de classificação módulo difeomorfismos de classe $C^{1}$. Motivados por esta observação, estaremos interessados em responder a seguinte pergunta:

Quando uma família de aplicações $F_{t}: \mathbb{R}^{n} \rightarrow \mathbb{R}^{n-1}$ quase-homogêneas define uma família de curvas quase-homogêneas $X_{t}=F_{t}^{-1}(0)$, pela origem de $\mathbb{R}^{n}$, bi-Lipschitz trivial?

Exemplo 2.4.2. Consideremos $F_{t}: \mathbb{R}^{3} \rightarrow \mathbb{R}^{2}$ dada por $F_{t}(x, y, z)=\left(z^{3}+t x, y^{3}-x^{2}\right)$. Temos que $X_{t}=F_{t}^{-1}(0)$ não define uma família, de curvas pela origem de $\mathbb{R}^{3}$, biLipschitz trivial. Com efeito, $X_{0}$ possui os seguintes semi-ramos

$$
\begin{aligned}
& X_{0}^{\mathrm{I}}=\left\{\left(r^{3}, r^{2}, 0\right): r \geqslant 0\right\} \\
& X_{0}^{2}=\left\{\left(-r^{3}, r^{2}, 0\right): r \geqslant 0\right\} .
\end{aligned}
$$

Portanto, $\operatorname{sh}\left(X_{0}^{1}, X_{0}^{2}\right)=\frac{3}{2}$. Por outro lado, para $t \neq 0, X_{t}$ possui os seguintes semiramos

$$
\begin{aligned}
& X_{t}^{1}=\left\{\left(-\frac{1}{t} r^{3}, r^{2}, r\right): r \geqslant 0\right\} \\
& X_{t}^{2}=\left\{\left(\frac{1}{t} r^{3}, r^{2}, r\right): r \geqslant 0\right\} .
\end{aligned}
$$

Portanto, $\operatorname{sh}\left(X_{t}^{1}, X_{t}^{2}\right)=3$. Observo que $F_{0}$ não satisfaz $\Sigma_{f} \cap f^{-1}(0)=\{0\}$. 
Exemplo 2.4.3. Consideremos $f_{t}: \mathbb{R}^{2} \rightarrow \mathbb{R}$ dada por $f_{t}(x, y)=x^{4}-y^{6}+t x^{2} y^{3}$, deformação quase-homogênea do germe $f:\left(\mathbb{R}^{2}, 0\right) \rightarrow(\mathbb{R}, 0)$ dado por $f(x, y)=x^{4}-y^{6}$. Vimos na seção anterior que $X_{t}=f_{t}^{-1}(0)$ define uma família de curvas, pela origem de $\mathbb{R}^{2}$, semi-algebricamente bi-Lipschtz trivial. Observo que $f$ tem singularidade isolada na origem.

Seja $X$ uma curva plana quase-homogênea com respeito aos pesos $\left(a_{1}, a_{2}\right)$, isto é, $X$ é invariante pela ação (2.4), e com semi-ramos reais $X=U_{i \in I} X_{i}$.

Lema 2.4.4. Se $a_{1}>a_{2} \geqslant 1$, então o cone tangente a $(X, 0)$ está contido nos eixos de coordenadas de $\mathbb{R}^{2}$.

Prova. Seja $S$ um semi-ramo de $X, 0$ e seja $(p, q)$ um ponto em $S$ distinto da origem. Como $X$ é quase-homogênea com respeito aos pesos $\left(a_{1}, a_{2}\right)$, podemos parametrizar $S$ por

$$
S=\left\{\left(s^{a_{1}} p, s^{a_{2}} q\right): s \geqslant 0\right\}
$$

Se $q \neq 0$, então o $y$-eixo é tangente a $S$, pois estamos supondo $a_{1}>a_{2}$. Caso contrário, claramente o $x$-eixo é tangente a $S$.

O lema acima mostra quão previsível é o comportamento das tangentes de famílias de curvas quase-homogêneas no caso em que os pesos são distintos. O lema seguinte é fundamental para o esclarecimento do comportmento bi-Lipschitz de curvas quasehomogêneas planas.

Lema 2.4.5. Para cada par de semi-ramos $X_{i}$ e $X_{j}$ de $(X, 0)$, com mesma semi-reta real tangente, $\operatorname{sh}\left(X_{i}, X_{j}\right)=\frac{a_{2}}{a_{1}}$.

Prova. No caso $a_{2}=a_{1}$, isto é, no caso homogêneo nada temos a provar. Então, suponhamos $a_{1}>a_{2}$. Como $X_{\imath}$ e $X_{j}$ são semi-ramos distintos de $(X, 0)$, pelo lema anterior temos que a semi-reta tangente real a $X_{i}$ e $X_{j}$ é gerada por $\pm e_{2}$, isto é, $(0, \pm 1)$. Sem perda de generalidade, suponhamos que tal semi-reta seja gerada por $(0,1)$. Sejam $p=\left(p_{1}, p_{2}\right) \in X_{i}$ e $q=\left(q_{1}, q_{2}\right) \in X_{j}$. Claramente, temos $p_{2} \neq 0$ e $q_{2} \neq 0$. Afirmamos que é possivel escolher $p$ e $q$ nas condições acima tais que 
$\frac{p_{1}}{\left(p_{2}\right)^{\frac{1}{a_{2}}}} \neq \frac{q_{1}}{\left(q_{2}\right)^{\frac{1}{a_{2}}}}$. Com efeito, se $\frac{p_{1}}{\left(p_{2}\right)^{\frac{1}{a_{2}}}}=\frac{q_{1}}{\left(g_{2}\right)^{\frac{1}{a_{2}}}}$, então troquemos $p$ por $p^{\prime}=t \cdot p$ com $t>0$ suficientemente pequeno. Obviamente, $p^{\prime} \in X_{i}$ e $p_{2}^{\prime} \neq 0$. Se tivéssemos $\frac{p_{1}^{\prime}}{\left(p_{2}^{\prime}\right)^{\frac{1}{a_{2}}}}=\frac{q_{1}}{\left(q_{2}\right)^{\frac{1}{a_{2}}}}$, então $t^{a_{1}-1}=1$. Como $a_{1}>a_{2} \geqslant 1$, e $t>0$ foi escolhido pequeno, temos uma contradição .

Usando que $X_{i}$ e $X_{j}$ são invariantes pela ação 2.4, concluímos o seguinte:

$$
X_{i}=\left\{\left(t^{a_{1}} p_{1}, t^{a_{2}} p_{2}\right): 0 \leqslant t\right\} \quad \text { e } \quad X_{j}=\left\{\left(t^{a_{1}} q_{1}, t^{a_{2}} q_{2}\right): 0 \leqslant t\right\} .
$$

Assim, se $x_{i}(r)$ é o ponto na interseção $X_{i} \cup S_{r}(0)$ e se $x_{j}(r)$ é o ponto na interseção $X_{j} \cup S_{r}(0)$, temos:

$$
x_{i}(r)=\left(r, r^{\frac{a_{1}}{a_{2}}} \frac{p_{2}}{\left|p_{1}\right|^{\frac{1}{a_{1}}}}\right) \quad \text { e } \quad x_{j}(r)=\left(r, r^{\frac{a_{1}}{a_{2}}} \frac{q_{2}}{\left|q_{1}\right|^{\frac{1}{a_{1}}}}\right) .
$$

Agora, como $\frac{p_{1}}{\left(p_{2}\right)^{\frac{1}{a_{2}}}} \neq \frac{q_{1}}{\left(q_{2}\right)^{\frac{1}{a_{2}}}}$, temos ord $\operatorname{or}_{r}\left\|x_{i}(r)-x_{j}(r)\right\|=\frac{a_{2}}{a_{1}}$.

Teorema 2.4.6. Seja $f_{t}: \mathbb{R}^{2} \rightarrow \mathbb{R}$ uma deformação quase-homegênea, com respeito aos pesos $a_{1} \geqslant a_{2} \geqslant 1$, de um germe $f$, definindo uma familia de curvas planas $X_{t}=f_{t}^{-1}(0)$. Se $f$ define um germe de codimensão finita na origem, então $\left(X_{t}, 0\right)_{t}$ é semi-algebricamente bi-Lipschitz trivial para $t$ pequeno.

Prova. Se $a_{1}=a_{2}$, nada temos a fazer. Então, podemos supor $a_{1}>a_{2}$. Suponhamos, primeiro, que $X_{0}$ não contenha o $y$-eixo. Nesse caso, para $t$ pequeno, $X_{t}$ não intersecta o $y$-eixo fora da origem. Portanto, para cada $t$ pequeno, vale: $X_{t}^{i}, X_{t}^{j}$ possuem a mesma semi-reta real tangente se, e somente se, $X_{0}^{i}, X_{0}^{j}$ possuem a mesma semi-reta real tangente. Assim, diante do lema acima, temos: $\operatorname{sh}\left(X_{t}^{i}, X_{t}^{j}\right) \equiv \operatorname{sh}\left(X_{0}^{i}, X_{0}^{j}\right)$.

Agora, suponhamos que $X_{0}$ contenha o $y$-eixo. Nesse caso, $X_{t}$ também contém o. $y$-eixo, pois o fato de $y x^{a} \in \operatorname{supp}(f)$ para algum $a$, implica que $x^{b} \notin \operatorname{supp}(f)$ para todo b. Assim,

$$
X_{t}^{1}=\{(s, 0): s \geqslant 0\} \text { e } X_{t}^{1}=\{(s, 0): s \leqslant 0\}
$$

são semi-ramos reais de $X_{t}$ para todo $t$ pequeno. Portanto

$$
\operatorname{sh}\left(X_{t}^{i}, X_{t}^{j}\right) \equiv \begin{cases}1 & \text { se } i \in\{1,2\} \quad \text { ou } j \in\{1,2\} \\ \frac{a_{2}}{a_{1}} & \text { caso contrário }\end{cases}
$$


De qualquer forma, temos $\operatorname{sh}\left(X_{t}^{i}, X_{t}^{j}\right) \equiv$ const.

Por outro lado, segue dos resultados apresentados em [9] que a família acima é topologicamente trivial. Então, do Teorema 2.3.15, segue-se o resultado.

Observação 2.4.7. No capítulo sobre curvas complexas, mostramos que deformações $\mu$-constantes $f_{t}: \mathbb{C}^{2} \rightarrow \mathbb{C}$ de $f$, em que $\mu$ é o número de Milnor de $f$, definem famílias de curvas bi-Lipschitz triviais.

Relembremos algo a respeito da determinação finita de germes de aplicaçōes analíticas. De acordo com [31], dado um germe de aplicaçāo analítica $F: \mathbb{R}^{n} \rightarrow \mathbb{R}^{p}$, temos que $\Sigma_{F} \cap F^{-1}(0)=0$ é uma condição necessária e suficiente para que $f$ seja $C^{l}-\mathcal{K}$-determinado para cada $0 \leqslant l<\infty$.

A partir do Teorema 2.4 .6 surge naturalmente a seguinte pergunta:

Se $F_{t}: \mathbb{R}^{n} \rightarrow \mathbb{R}^{n-1}$ é uma deformação quase-homogênea de uma aplicação quasehomogênea $F: \mathbb{R}^{n} \rightarrow \mathbb{R}^{n-1}$ com respeito aos pesos $a_{1}>\cdots>a_{n}>1$ satisfazendo $\Sigma_{F} \cap F^{-1}(0)=0$, então $F_{t}$ define uma família de curvas $X_{t}=F^{-1}(0)$ bi-Lipschitz trivial ?

O seguinte exemplo responde negativamente a essa pergunta.

Exemplo 2.4.8. $F_{t}: \mathbb{R}^{3} \rightarrow \mathbb{R}^{2}$ dada por $F(x, y, z)=\left(y+t z^{2}, x^{4}-z^{12}\right)$ 


\section{Capítulo 3}

\section{Teoria métrica de curvas complexas}

Em [8], E. Brieskorn observa que, se esperamos capturar informações a respeito de propriedades qualitativas locais de curvas analíticas complexas em $\mathbb{C}^{2}$, não devemos abordá-las de forma abstrata. Essa afirmação é justificada pelo resultado abaixo Proposição 3.0.9. Todo ramo de curva analítica em $\mathbb{C}^{2}$ é homeomorfo a $(\mathbb{C}, 0)$.

Este resultado justifica a seguinte relação de equivalência topológica, entre germes de subconjuntos analíticos em $\mathbb{C}^{d}$.

Definição 3.0.10. Sejam $C$ e $D$ subconjuntos analíticos de $\mathbb{C}^{d}$ e sejam $x \in C$, $y \in D$. O germe $(C, x)$ é topologicamente equivalente a $(D, y)$ quando existe um homeomorfismo $F:\left(\mathbb{C}^{2}, x\right) \rightarrow\left(\mathbb{C}^{2}, y\right)$ tal que $F(C)=D$.

Claramente, a abordagem acima é mais fina do que a abordagem abstrata, no sentido que: se $(C, x)$ é topologicamente equivalente a $(D, y)$, então $(C, x)$ é homeomorfo a $(D, y)$. De fato, essa abordagem é estritamente mais fina do que a abstrata, por exemplo, temos que o germe de curva complexa $(C, 0)$, definido por $C: z^{2}=w^{3}$, é homeomorfo mas não é topologicamente equivalente à reta $(\mathbb{C}, 0)$.

A classificação proposta acima foi essencialmente analisada a partir dos anos 20 do século passado. Os matemáticos K. Brauner, K. Kähler, W. Burau, O. Zariski foram responsáveis pela solução desse problema. Um bom apanhado das técnicas e resultados obtidos a respeito desse tema pode ser encontrado em [8]. Uma solução completa para o problema de classificação proposto na Definição 3.0.10 é o seguinte 
Teorema 3.0.11. Dois germes de curvas complexas são topologicamente equivalentes se, e somente se, existe uma bijeção entre seus ramos que preserva seqüência de expoentes característicos e indices de interseção de pares de ramos.

Aqui, propomo-nos analisar germes de curvas complexas sob o ponto de vista métrico. Sendo mais específicos, consideramos germes de curvas complexas módulo homeomorfismos bi-Lipschitz. A princípio, temos bem definidas duas métricas naturais sobre subconjuntos subanalíticos de espaços euclidianos. A saber, temos a métrica euclidiana induzida e a métrica geodésica que é intrínseca do subconjunto. Por analogia à Proposição 3.0.9, apresentamos a seguinte proposição para justificar a nossa decisão por estudar tais conjuntos como subespaços métricos de espaços euclidianos.

Proposição 3.0.12. Para qualquer ramo de curva analítica complexa $(C, x)$ em $\mathbb{C}^{2}$, munido da métrica geodésica intrínseca, existe um germe de aplicação subanalítica bi-Lipschitz $F:(C, x) \rightarrow(\mathbb{C}, 0)$.

Em [3], classificamos completamente os germes de conjuntos semi-algébricos de dimensão real 1, equipados da métrica euclidiana induzida, módulo homeomorfismos bi-Lipschitz. Em [2], L. Birbrair classifica completamente os germes de conjuntos semi-algébricos de dimensāo real 2, equipados da métrica geodésica intrínseca, módulo homeomorfismos bi-Lipschitz. L. Birbrair aponta para a dificuldade de se obter uma classificação dos mesmos germes acima quando vistos como subespaços euclidianos. Aqui, consideramos a métrica euclidiana induzida e, neste contexto, apresentaremos uma classificação completa dos germes de curvas (complexas) analíticas planas com singularidade isolada. De fato, provamos o seguinte

Teorema 3.0.13. Sejam $(X, 0)$ e $(\widetilde{X}, 0)$ germes de curvas analíticas complexas planas. Existe um germe de aplicaçäo subanalítica bi-Lipschitz $F:(X, 0) \rightarrow(\widetilde{X}, 0)$ se, e somente se, existe uma bijeção entre os ramos desses germes que preserva seqüência de expoentes característicos $e$ indices de interseção de pares de ramos.

Vale observar que a classe de germes que consideramos aqui não é formada por germes normalmente mergulhados em $\mathbb{R}^{4}$ (cf [4]) e, portanto, a classificação que obtemos não vem como conseqüência dos resultados apresentados em [2]. 
Os resultados deste capítulo compõem o artigo [11] que foi submetido para publicação em novembro de 2001.

\subsection{Arcos de Teste}

Nesta seção, relembramos o conceito de semi-complexo de Hölder de germes de curvas reais, apresentamos o conceito de arcos de teste e demonstramos um lema de grande importância para este capítulo.

Dado um germe de subconjunto semi-analítico $(X, x)$, em $\mathbb{R}^{p}$, de dimensão real 1 , equipado com a métrica euclidiana induzida, para cada par de semi-ramos $X_{i}, X_{j}$, de $(X, x)$, temos o seguinte número:

$$
\operatorname{sh}\left(X_{i}, X_{j}\right)=\operatorname{ord}_{r}\left[\operatorname{dist}\left(X_{i} \cap S_{r}(x), X_{j} \cap S_{r}(x)\right)\right]
$$

As idéias discutidas em [3] são de fundamental importância neste capítulo, pois estas esclarecem o comportamento métrico de germes de conjuntos semi-analíticos de dimensão real 1 e o conceito de arco de teste, o qual aparece de forma contundente aqui.

Definição 3.1.1. Um arco de teste é um germe de conjunto semi-analítico de dimensão real 1, munido da métrica induzida, com apenas um semi-ramo real.

Lema 3.1.2. Sejam $\left(\Gamma_{1}, x\right)$ e $\left(\Gamma_{2}, x\right)$ arcos de teste e $F:\left(\Gamma_{1} \cup \Gamma_{2}, x\right) \rightarrow \mathbb{R}^{p}$ um germe de aplicação subanalítica tal que sua restrição a cada arco de teste $\Gamma_{\imath}$ é uma aplicação Lipschitz, $i=1,2$. Se

$$
\left|F\left(x_{1}(r)\right)-F\left(x_{2}(r)\right)\right| \leqslant\left|x_{1}(r)-x_{2}(r)\right|
$$

em que $\left\{x_{i}(r)\right\}=S_{r}(x) \cap \Gamma_{i}$, então $F$ é um germe de aplicação Lipschitz.

Prova. Admitamos todas as hipóteses do lema e suponhamos, por absurdo, que a sua conclusão seja falsa. Então, pelo Lema de Seleção da Curva, existem curvas analíticas $q_{1}, q_{2} ;[0, \delta) \rightarrow \Gamma$ tais que $q_{i}(0)=0, q_{i}(s) \in \Gamma, \forall s, i=1,2 \mathrm{e}$

$$
\left|F\left(q_{1}(r)\right)-F\left(q_{2}(r)\right)\right| \gg\left|q_{1}(r)-q_{2}(r)\right| .
$$


Podemos supor que $\left|q_{1}(s)\right| \geqslant\left|q_{2}(s)\right|, \forall s$, pois caso contrário, por analiticidade, teríamos uma contradição com uma das hipóteses do lema acima. Tómando uma reparametrização, caso seja necessário, podemos supor que $\left|q_{1}(s)\right|$ e $\left|q_{2}(s)\right|$ sāo séries de potências fracionárias e $\left|q_{1}(s)\right| \geqslant\left|q_{2}(s)\right|=s$, para cada $s$. Assim, usando as hipóteses do lema, temos

$$
\begin{aligned}
\left|q_{1}(s)-q_{2}(s)\right| & \ll\left|F\left(q_{1}(s)\right)-F\left(x_{1}(s)\right)\right|+\left|F\left(x_{1}(s)\right)-F\left(q_{2}(s)\right)\right| \\
& \lesssim\left|q_{1}(s)-x_{1}(s)\right|+\left|x_{1}(s)-q_{2}(s)\right|
\end{aligned}
$$

Pelo Lema 2.1.1, temos

$$
\left|x_{1}(s)-q_{2}(s)\right| \lesssim\left|q_{1}(s)-q_{2}(s)\right|
$$

Portanto,

$$
\left|q_{1}(s)-q_{2}(s)\right| \ll\left|q_{1}(s)-x_{1}(s)\right| .
$$

E, pela desigualdade triangular, temos a seguinte contradição :

$$
\left|q_{1}(s)-q_{2}(s)\right| \approx\left|q_{1}(s)-x_{1}(s)\right|
$$

\subsection{Caso irredutível}

Seja $(C, 0)$ um germe de curva analiticamente irredutível em $\mathbb{C}^{2}$ (Ramo), a menos de uma mudança analítica de coordenadas, podemos supor que $(C, 0)$ tem uma parametrização do tipo abaixo:

$$
\left\{\begin{array}{c}
x=t^{m} \\
y=t^{n}+a_{1} t^{n_{1}}+\ldots,
\end{array}\right.
$$

em que $m$ é a multiplicidade de $(C, 0), m$ não divide o inteiro $n$ e $y(t) \in \mathbb{C}\{t\}$. A série de potências fracionárias $y\left(x^{\frac{1}{m}}\right)$ é conhecida como Parametrização de NewtonPuiseux de $(C, 0)$ e todas as outras parametrizaçōes de Newton-Puiseux de $(C, 0)$ 
são obtidas da parametrização acima via $x^{\frac{1}{m}} \mapsto w x^{\frac{1}{m}}$ em que $w$ é raiz $m$-ésima da unidade.

Observação 3.2.1. Quando falarmos em aplicação bi-Lipschitz, salvo mencionado contrário, estaremos considerando os espaços métricos envolvidos na questão munidos da métrica euclidiana induzida.

Exemplo 3.2.2 (Fundamental). Sejam $C: w^{2}=z^{3}$ e $D: w^{2}=z^{5}$. Então não existe um germe de aplicaçāo subanalítica bi-Lipschitz $F:(C, 0) \rightarrow(D, 0)$.

Prova. Consideremos $\Sigma_{1}, \Sigma_{2}, \Sigma_{3}, \Sigma_{4}$ os seguintes semi-ramos em $D$ :

$$
\Sigma_{1}=\left\{\left(r, r^{\frac{5}{2}}\right): r \geqslant 0\right\} \quad \Sigma_{2}=\left\{\left(r i, r^{\frac{5}{2}} e^{\frac{i \pi}{4}}\right): r \geqslant 0\right\}
$$

e

$$
\Sigma_{3}=\left\{\left(r,-r^{\frac{5}{2}}\right): r \geqslant 0\right\} \quad \Sigma_{4}=\left\{\left(-r i, r^{\frac{5}{2}} e^{\frac{i 3 \pi}{4}}\right): r \geqslant 0\right\}
$$

Consideremos agora:

$$
\Gamma_{k}(r)=\left(r e^{i \gamma_{k}(r)}, r^{\frac{3}{2}} e^{i \frac{3 \gamma_{k}(r)}{2}}\right) \in S_{r}(0) \cap F\left(\Sigma_{k}\right) ; k=1,2,3,4
$$

Decorre do Teorema 2.1.3 que

$$
\left\|\Gamma_{1}(r)-\Gamma_{3}(r)\right\| \approx r^{\frac{5}{2}}
$$

Daí, podemos escolher $\gamma_{1}, \gamma_{3}$ tais que

$$
\lim _{r \rightarrow 0}\left(\gamma_{1}(r)-\gamma_{3}(r)\right)=4 k \pi ; \quad k=0 \quad \text { ou } \quad 1
$$

Suponhamos

$$
\lim _{r \rightarrow 0}\left(\gamma_{1}(r)-\gamma_{3}(r)\right)=0
$$

Nesse caso, temos que ocorre uma das seguintes alternativas

$$
\lim _{r \rightarrow 0}\left(\gamma_{1}(r)-\gamma_{2}(r)\right)=0 \quad \text { ou } \quad \lim _{r \rightarrow 0}\left(\gamma_{1}(r)-\gamma_{4}(r)\right)=0
$$

Suponhamos que

$$
\lim _{r \rightarrow 0}\left(\gamma_{1}(r)-\gamma_{2}(r)\right)=0
$$


Em particular,

$$
\left\|\Gamma_{1}(r)-\Gamma_{2}(r)\right\| \ll r
$$

Denotemos $\delta_{k}(r)=F^{-1}\left(\Gamma_{k}(r)\right) ; k=1$, 2. Então, temos

$$
\delta_{1}(r)=\left(f(r), f(r)^{\frac{5}{2}}\right) \quad \text { e } \quad \delta_{2}(r)=\left(g(r) i, g(r)^{\frac{5}{2}} e^{\frac{i \pi}{4}}\right)
$$

em que

$$
|f(r)| \approx|g(r)| \approx r
$$

E, daí,

$$
\left\|\delta_{1}(r)-\delta_{2}(r)\right\| \gtrsim|f(r)-i g(r)| \approx r .
$$

Por outro lado, como $F$ é bi-Lipschitz,

$$
\left\|\delta_{1}(r)-\delta_{2}(r)\right\| \approx\left\|\Gamma_{1}(r)-\Gamma_{2}(r)\right\|
$$

o que é um absurdo.

Observo que os outros casos são analisados de forma completamente análoga.

Observação 3.2.3. A prova exibida acima pode ser adaptada, sem o menor esforço para mostrar que não existe homeomorfismo bi-Lipschitz subanalítiço entre os seguintes germes de curvas complexas planas:

$$
C: w^{2}=z^{2 k+1} \quad \text { e } \quad D: w^{2}=z^{2 l+1}
$$

quando $k \neq l$.

Teorema 3.2.4. Dados $(C, 0)$ e $(\tilde{C}, 0)$ dois germes de curvas analiticamente irredutiveis em $\mathbb{C}^{2}$, temos que existe $F:(C, 0) \rightarrow(\tilde{C}, 0)$ germe de aplicação subanalítica bi-Lipschitz se, e somente se, $\beta(C)=\beta(\tilde{C})$.

Apresentaremos uma demonstração do teorema acima, que tem seu cerne completamente explicitado no Exemplo 3.2.2, embora as idéias apresentadas lá não resolvam este problema de uma forma imediata. 
A seguir, analisaremos o comportamento métrico de pares de arcos de teste em um ramo analítico.

Seja, $(C, 0)$ um ramo com multiplicidade $n$ e pares característicos $\left(m_{1}, n_{1}\right), \ldots,\left(m_{g}, n_{g}\right)$. Sejam $\Gamma_{1}, \Gamma_{2}$ arcos de teste em $(C, 0)$ descritos por

$$
\Gamma_{j}(r)=\left(r e^{i \alpha_{j}(r)}, y\left(r^{\frac{1}{n}} e^{i \frac{\alpha_{j}(r)}{n}}\right)\right) \in S_{r}(0) \cap \Gamma_{j}, \quad j=1,2
$$

em que $y\left(x^{\frac{1}{n}}\right)$ é uma parametrização de Newton-Puiseux de $(C, 0)$ e $\alpha_{1}, \alpha_{2}$ são funções ângulo tomando valores no intervalo $[0,2 n \pi]$. Sejam $g(r)=\left\|\Gamma_{1}(r)-\Gamma_{2}(r)\right\|$ e $h(r)=r\left\|e^{i \alpha_{1}(r)}-e^{i \alpha_{2}(r)}\right\|$. Então, vale o seguinte

Lema 3.2.5. Se

$$
\frac{1}{2 \pi} \lim _{r \rightarrow 0}\left(\alpha_{1}(r)-\alpha_{2}(r)\right)=k \in \mathbb{Z}
$$

entäo ord $\operatorname{ord}_{r}(g)=\min \left\{\operatorname{ord}_{r}(h), \frac{m_{j}}{n_{1} \ldots n_{j}}\right\}$ em que $j=\min \left\{i: \quad \frac{k}{n_{1} \ldots n_{i}} \notin \mathbb{Z}\right\}$.

Prova. É suficiente observarmos a equação (1.3).

Observação 3.2.6. É claro que, se

$$
\frac{1}{2 \pi} \lim _{r \rightarrow 0}\left(\alpha_{1}(r)-\alpha_{2}(r)\right) \notin \mathbb{Z},
$$

então $\operatorname{ord}_{r}(g)=1$

Com essas observações, já podemos concluir que se dois ramos $(C, \dot{0})$ e $(D, 0)$ possuem os mesmos expoentes característicos, então existe um germe de aplicação subanalítica bi-Lipschitz $F:(C, 0) \rightarrow(D, 0)$. Com efeito, decorre imediatamente dos lemas 3.1 .2 e 3.2 .5 que

$$
F\left(t^{n}, y(t)\right)=\left(t^{n}, z(t)\right)
$$

define um germe de aplicação subanalítica bi-Lipschitz de $(C, 0)$ sobre $(D, 0)$, em que $y\left(x^{\frac{1}{n}}\right)$ e $z\left(x^{\frac{1}{n}}\right)$ são parametrizações de Newton-Puiseux de $(C, 0)$ e $(D, 0)$ respectivamente.

Reciprocamente, suponhamos que $F:(C, 0) \rightarrow(D, 0)$ seja um germe de aplicação subanalítica bi-Lipschitz e sejam $y\left(x^{\frac{1}{n}}\right)$ e $z\left(x^{\frac{1}{n}}\right)$ parametrizações de NewtonPuiseux de $(C, 0)$ e $(D, 0)$ respectivamente. Sejam $n=\operatorname{multiplicidade}(C, 0)$ e $\tilde{n}=$ multiplicidade $(D, 0)$. 
Lema 3.2.7. Sejam $\Sigma_{1}, \Sigma_{2}$ arcos de teste em $(C, 0)$ descritos por

$$
\Sigma_{j}(r)=\left(r e^{i \sigma_{j}(r)}, y\left(r^{\frac{1}{n}} e^{i \frac{\sigma_{j}(r)}{n}}\right)\right) \in S_{r}(0) \cap \Sigma_{j}, \quad j=1,2
$$

Es sejam $\Gamma_{1}=F\left(\Sigma_{1}\right), \Gamma_{2}=F\left(\Sigma_{2}\right)$ arcos de teste em $(D, 0)$ descritos por

$$
\Gamma_{j}(r)=\left(r e^{i \gamma_{j}(r)}, z\left(r^{\frac{1}{\tilde{n}}} e^{i \frac{\gamma_{j}(r)}{\tilde{n}}}\right)\right) \in S_{r}(0) \cap \Sigma_{j}, \quad j=1,2
$$

Então

$$
\frac{1}{2 \pi} \lim _{r \rightarrow 0}\left(\sigma_{1}(r)-\sigma_{2}(r)\right) \in n \mathbb{Z}
$$

se, e somente se,

$$
\frac{1}{2 \pi} \lim _{r \rightarrow 0}\left(\gamma_{1}(r)-\gamma_{2}(r)\right) \in \tilde{n} \mathbb{Z}
$$

Prova. Suponhamos que

$$
\frac{1}{2 \pi} \lim _{r \rightarrow 0}\left(\sigma_{1}(r)-\sigma_{2}(r)\right)=k n ; k \in \mathbb{Z}
$$

Nesse caso, $\Sigma_{1}$ e $\Sigma_{2}$ definem dois triângulos complementares na superfície subanalítica real $(C, 0)$ cujos bordos são coincidentes e iguais a $\Sigma_{1} \cup \Sigma_{2}$, mais ainda, um desses triângulos somente admite arcos de teste tangentes aos arcos $\Sigma_{1}$ e $\Sigma_{2}$. Por outro lado, se

$$
\frac{1}{2 \pi} \lim _{r \rightarrow 0}\left(\gamma_{1}(r)-\gamma_{2}(r)\right)=\alpha \notin \tilde{n} \mathbb{Z}
$$

também temos dois triângulos complementares na superfície subanalítica real $(D, 0)$ cujos bordos são $\Gamma_{1} \cup \Gamma_{2}$ mas, neste caso, os dois triângulos admitem arcos de teste não tangentes a $\Gamma_{1}$ e $\Gamma_{2}$ e, portanto, temos uma contradição ao fato de $F$ ser subanalítica. bi-Lipschitz.

Proposição 3.2.8. $n=\tilde{n}$.

Prova. Suponhamos, por absurdo, que $n>\tilde{n}$ e consideremos $\alpha_{j}=2 j \pi$, com $j=0, \ldots, n-1$. Seja $\Sigma_{j}$ o semi-ramo real em $(C, 0)$ descrito por

$$
\Sigma_{j}(r)=\left(r e^{i \alpha_{j}}, y\left(r^{\frac{1}{n}} e^{i \frac{\alpha_{j}}{n}}\right)\right) \in S_{r}(0) \cap \Sigma_{j}, \quad j=0, \ldots, n-1
$$


Seja $\widetilde{\Sigma_{j}}=F\left(\Sigma_{j}\right)$ descrito por

$$
\widetilde{\Sigma}_{j}(r)=\left(r e^{i \sigma_{j}(\tau)}, y\left(r^{\frac{2}{\bar{n}}} e^{i \frac{\sigma_{j}(r)}{\tilde{n}}}\right)\right) \in S_{r}(0) \cap \widetilde{\Sigma_{j}}, \quad j=0, \ldots, n-1
$$

$\operatorname{com} \sigma_{0}(r) \leqslant \ldots \leqslant \sigma_{n-1}(r) \leqslant \sigma_{0}(r)+2 \tilde{n} \pi$

Observemos que, pelo Lema 3.2.7, não é possível termos

$$
\frac{1}{2 \pi} \lim _{r \rightarrow 0}\left(\sigma_{n-1}(r)-\sigma_{0}(r)\right) \in \tilde{n} \mathbb{Z}
$$

Então, pelo Princípio das Gavetas, temos que existe $j$ tal que

$$
\lim _{r \rightarrow 0}\left(\sigma_{j+1}(r)-\sigma_{j}(r)\right)=0
$$

E, novamente, utilizamos o Lema 3.2.7, para afirmar que isto é um absurdo.

Sejam $\left(m_{1}, n_{1}\right), \ldots,\left(m_{g}, n_{g}\right)$ os pares característicos de $(C, 0)$ e $\left(p_{1}, q_{1}\right), \ldots,\left(p_{\bar{g}}, q_{\bar{g}}\right)$ os pares característicos de $(D, 0)$. Vale observar que $n_{1} \ldots n_{g}=n=q_{1} \ldots q_{\bar{g}}$ (vide lema acima).

Proposição 3.2.9. $m_{g}=p_{\tilde{g}}$

Prova. Suponhamos, por absurdo, que $m_{g}>p_{\tilde{g}}$. Sejam $\Gamma_{0}, \Gamma_{1}$ os semi-ramos reais em $(C, 0)$ descritos por

$$
\Gamma_{0}(r)=\left(r, y\left(r^{\frac{1}{n}}\right)\right) \quad \Gamma_{1}(r)=\left(r, y\left(r^{\frac{1}{n}} e^{\frac{2 n_{1} \cdots n_{g-1} \pi}{n}}\right)\right)
$$

Claramente, temos ord ${ }_{r}\left\|\Gamma_{0}(r)-\Gamma_{1}(r)\right\|=\frac{m_{g}}{n}$. Consideremos $\Sigma_{j}=F\left(\Gamma_{j}\right) ; \quad j=0,1$, semi-ramos reais em $(D, 0)$ descritos por

$$
\Sigma_{j}(r)=\left(r e^{i \alpha_{j}(r)}, z\left(r^{\frac{1}{n}} e^{i \frac{\alpha_{j}(r)}{n}}\right)\right) \in S_{r}(0) \cap \Sigma_{j}, \quad j=0,1
$$

Como $F$ é bi-Lipschitz, temos $\operatorname{ord}_{r}\left\|\Sigma_{0}(r)-\Sigma_{1}(r)\right\|=\frac{m_{g}}{n}$. Assim, pelo Lema 3.2.5, temos que

$$
\frac{1}{2 n \pi} \lim _{r \rightarrow 0}\left(\alpha_{0}(r)-\alpha_{1}(r)\right) \in \mathbb{Z}
$$

Mas, isto contradiz o Lema 3.2.7. 
Proposição 3.2.10. $n_{g}=q_{\tilde{g}}$

Prova. Suponhamos, por absurdo, que $n_{g}>q_{\tilde{g}}$. Sejam $\gamma_{j}=2 j n_{1} \ldots n_{g-1} \pi ; j=$ $1, \ldots, n_{g}$ e $\Gamma_{j}$ semi-ramo real em $(C, 0)$ descrito por

$$
\Gamma_{j}(r)=\left(r, y\left(r^{\frac{1}{n} e^{i \frac{2 j n_{1} \ldots n_{g-1} \pi}{n}}}\right)\right), \quad j=1, \ldots, n_{g}
$$

Claramente, temos $\operatorname{ord}_{r}\left\|\Gamma_{j}(r)-\Gamma_{j+1}(r)\right\|>1$. Consideremos $\Sigma_{j}=F\left(\Gamma_{j}\right) ; j=$ $1, \ldots, n_{g}$, semi-ramos reais em $(D, 0)$ descritos por

$$
\Sigma_{j}(r)=\left(r e^{i \sigma_{j}(r)}, z\left(r^{\frac{1}{n}} e^{i \frac{\sigma_{j}(r)}{n}}\right)\right) \in S_{r}(0) \cap \Sigma_{j}, \quad j=1, \ldots, n_{g}
$$

$\operatorname{com} \sigma_{0}(r) \leqslant \ldots \leqslant \sigma_{n-1}(r) \leqslant \sigma_{0}(r)+2 n \pi$.

Sendo $n_{g}>q_{\tilde{g}}$ e ord ${ }_{r}\left\|\Gamma_{j}(r)-\Gamma_{j+1}(r)\right\|>1$, podemos garantir, a partir do Princípio das Gavetas, que existe $j$ tal que

$$
\lim _{r \rightarrow 0}\left(\sigma_{j}(r)-\sigma_{j+1}(r)\right)=0
$$

Agora, observamos que a última equação contradiz o Lema 3.2.7.

Observemos que os argumentos usados para demonstrar a proposição acima, são suficientes para mostrarmos que $g=\vec{g} \quad$ e $n_{i}=q_{i}, \forall i$. Com efeito, já provamos que valem as seguinte igualdades:

$$
m(C)=n=m(D), \quad m_{g}=p_{\bar{g}} \quad \text { e } \quad n_{g}=q_{\tilde{g}}
$$

Suponhamos, por absurdo, que $n_{g-1}>q_{\bar{g}-1}$. Agora, tomamos $n_{g-1} n_{g}$ pontos no intervalo $\left[2 n_{1} \ldots n_{g-2} \pi, 2 n \pi\right]$, a saber, $\gamma_{j}=2 j n_{1} \ldots n_{g-2} \pi ; j=1, \ldots, n_{g-1}+n_{g}$ e. arcos de teste em $(C, 0)$, digamos, $\Gamma_{1}, \ldots, \Gamma_{n_{g-1}+n_{g}}$ descritos por:

$$
\Gamma_{j}(r)=\left(r, y\left(r^{\frac{1}{n}} e^{i \frac{\gamma_{j}}{n}}\right)\right) \in S_{r}(0) \cap \Gamma_{j} ; \quad j=1, \ldots, n_{g-1} n_{g}
$$

Seja $\Sigma_{j}=F\left(\Gamma_{j}\right)$, arco de teste em $(D, 0)$ descrito por

$$
\Sigma_{j}(r)=\left(r e^{i \sigma_{j}(r)}, z\left(r^{\frac{1}{n}} e^{i \frac{\sigma_{j}(r)}{n}}\right)\right) \in S_{r}(0) \cap \Sigma_{j} ; \quad j=1, \ldots, n_{g-1} n_{g}
$$


Fazendo uso dos seguintes fatos: Lema 3.2.5, ord ${ }_{r}\left\|\Gamma_{j}(r)-\Gamma_{j+1}(r)\right\|>1$ e Princípio das Gavetas, temos que existe um $j$ tal que

$$
\lim _{r \rightarrow 0}\left(\sigma_{j+1}(r)-\sigma_{j}(r)\right)=0
$$

$E$, finalmente, o argumento que usamos para concluir que isso gera uma contradição é o Lema 3.2.7. Então, fica claro que com um processo de indução provamos que $g=\tilde{g} \quad$ e $\quad n_{i}=q_{i}, \forall i$.

Agora, para concluir que $\beta(C)=\beta(\widetilde{C})$ é suficiente mostrar que $m_{i}=p_{i} ; \forall i$. Para isso, tomemos $j$ como o menor índice tal que $m_{j} \neq p_{j}$. Mostremos primeiro que $j>1$. Por absurdo, suponhamos que $m_{1}>p_{1}$. Sejam $\Gamma_{0}, \Gamma_{1}$ os semi-ramos reais de $(C, 0)$ descritos por:

$$
\Gamma_{0}(r)=\left(r, y\left(r^{\frac{1}{n}}\right)\right) \quad \text { e } \quad \Gamma_{1}(r)=\left(r, y\left(r^{\frac{2}{n}} e^{\frac{2 i \pi}{n}}\right)\right)
$$

Como ord ${ }_{r}\left\|\Gamma_{0}(r)-\Gamma_{1}(r)\right\|=\frac{m_{1}}{n_{1}}$, podemos lançar mão do Lema 3.2 .5 para garantir que $n_{1}$ divide o inteiro

$$
\frac{1}{2 \pi} \lim _{r \rightarrow 0}\left(\sigma_{1}(r)-\sigma_{1}(r)\right)
$$

em que $\sigma_{j}(r)$ descreve o semi-ramo $\Sigma_{j}=F\left(\Gamma_{j}\right)$, isto é,

$$
\Sigma_{j}(r)=\left(r e^{i \sigma_{j}(r)}, z\left(r^{\frac{2}{n}} e^{i \frac{\sigma_{j}(r)}{n}}\right)\right) \in S_{r}(0) \cap \Sigma_{j}, \quad j=0,1
$$

Sabemos que não é possivel que o limite acima seja um inteiro múltiplo de $n$. Então, para $r>0$ suficientemente pequeno, vale

$$
\sigma_{0}(r) \leqslant \sigma_{2}(r) \leqslant \sigma_{1}(r) \quad \text { em que } \quad \sigma_{2}(r)=\sigma_{0}(r)+2 \pi
$$

Claramente, se definimos $\Sigma_{2}$ como sendo o semi-ramo real de $(D, 0)$ descrito por -

$$
\Sigma_{2}(r)=\left(r e^{i \sigma_{2}(r)}, z\left(r^{\frac{2}{n}} e^{i \frac{\sigma_{2}(r)}{n}}\right)\right) \in S_{r}(0) \cap \Sigma_{2}
$$

então $\operatorname{sh}\left(\Sigma_{0} \cup \Sigma_{2}, 0\right)>1$. Daí, pelo Teorema 2.1.3, temos $\operatorname{sh}\left(\Gamma_{0} \cup \Gamma_{2}, 0\right)>1$, em que $\Gamma_{2}$ é o semi-ramo real $\Gamma_{2}=F^{-1}\left(\Gamma_{2}\right)$, em $(C, 0)$, descrito por

$$
\Gamma_{2}(r)=\left(r e^{i \gamma_{2}(r)}, y\left(r^{\frac{1}{n}} e^{i \frac{\gamma_{2}(r)}{n}}\right)\right) \in S_{r}(0) \cap \Gamma_{2}
$$


$0 \leqslant \gamma_{2}(r) \leqslant 2 \pi$. Como $\operatorname{sh}\left(\Gamma_{0} \cup \Gamma_{2}, 0\right)>1$ e $0 \leqslant \gamma_{2}(r) \leqslant 2 \pi$, devemos ter

$$
\lim _{r \rightarrow 0} \gamma_{2}(r)=0 \text { ou } 2 \pi
$$

Mas, isto contradiz o fato dos seguintes inteiros

$$
\frac{1}{2 \pi} \lim _{r \rightarrow 0}\left(\alpha_{2}(0)-\alpha_{0}(r)\right) \quad \text { e } \frac{1}{2 \pi} \lim _{r \rightarrow 0}\left(\alpha_{1}(0)-\alpha_{2}(r)\right)
$$

não serem múltiplos de $n$.

Então, finalmente, podemos afirmar que $j>1$. Observo que já sabemos que vale a desigualdade $j<g$. Suponhamos por absurdo que $m_{j}>p_{j}$. Consideremos $\gamma_{0}=2 n_{1} \ldots n_{j-1} \pi, \gamma_{1}=2 \gamma_{0}$ e $\Gamma_{i}$ semi-ramo real de $(C, 0)$ descrito por

$$
\Gamma_{i}(r)=\left(r, y\left(r^{\frac{1}{n}} e^{\frac{i \gamma_{i}}{n}}\right)\right) \in S_{r}(0) \cap \Gamma_{i} ; \quad i=0,1
$$

Seja $\Sigma_{i}=F\left(\Gamma_{i}\right)$ semi-ramo real em $(D, 0)$ descrito por

$$
\Sigma_{i}(r)=\left(r e^{i \sigma_{i}(r)}, z\left(r^{\frac{1}{n}} e^{i \frac{\sigma_{i}(r)}{n}}\right)\right) \in S_{r}(0) \cap \Sigma_{i}, \quad i=0,1
$$

Como $\left\|\Gamma_{1}(r)-\Gamma_{0}(r)\right\|=\frac{m_{j}}{n_{1} \ldots n_{j}}$, podemos, a partir do Lema 3.2 .5 , garantir que $n_{1} \ldots n_{j}$ divide o inteiro

$$
\frac{1}{2 \pi} \lim _{r \rightarrow 0}\left(\sigma_{1}(r)-\sigma_{0}(r)\right)
$$

e, já sabemos que o inteiro acima não pode ser múltiplo de $n$. Portanto, para $r>0$ suficientemente pequeno, valem a seguintes desigualdades:

$$
\sigma_{0}(r) \leqslant \sigma_{2}(r) \leqslant \sigma_{1}(r) \quad \text { em que } \sigma_{2}(r)=\sigma_{0}(r)+2 n_{1} \ldots n_{j-1} \pi
$$

Temos, claramente,

$$
\operatorname{sh}\left(\Sigma_{0}, \Sigma_{2}\right)=\frac{p_{j}}{n_{1} \ldots n_{j}}=\operatorname{sh}\left(\Sigma_{1}, \Sigma_{2}\right)
$$

e, portanto,

$$
\operatorname{sh}\left(\Gamma_{0}, \Gamma_{2}\right)=\frac{p_{j}}{n_{1} \ldots n_{j}}=\operatorname{sh}\left(\Gamma_{1}, \Gamma_{2}\right)
$$

em que $\Gamma_{2}=F^{-1}\left(\Sigma_{2}\right)$. Donde, se

$$
\Gamma_{2}(r)=\left(r e^{i \gamma_{2}(r)}, y\left(r^{\frac{1}{n}} e^{i \frac{\gamma_{2}(r)}{n}}\right)\right) \in S_{r}(0) \cap \Gamma_{2}
$$


descreve $\Gamma_{2}$, então

$$
\frac{1}{2 \pi} \lim _{r \rightarrow 0}\left(\gamma_{i}(r)-\gamma_{2}(r)\right) \in \mathbb{Z}
$$

e $n_{1} \ldots n_{j-1}$ divide o inteiro acima. Com efeito, caso não ocorresse a divisão afirmada, teríamos pelo Lema 3.2 .5

$$
\begin{aligned}
\frac{p_{j}}{n_{1} \ldots n_{j}} & =\operatorname{sh}\left(\Gamma_{i}, \Gamma_{2}\right) \\
& =\min \left\{\operatorname{ord}_{r} r\left|e^{i \gamma_{i}(r)}-e^{i \gamma_{2}(r)}\right|, \frac{m_{j-1}}{n_{1} \ldots n_{j-1}}\right\} \\
& =\frac{p_{j-1}}{n_{1} \ldots n_{j-1}}, \quad i=0,1
\end{aligned}
$$

o que é um absurdo.

Como, $\gamma_{0}(r) \leqslant \gamma_{2}(r) \leqslant \gamma_{1}(r)$ e $\left|\gamma_{0}(r)-\gamma_{1}(r)\right|=2 n_{1} \ldots n_{j-1} \pi$, devemos ter

$$
\lim _{r \rightarrow 0}\left(\gamma_{2}(r)-\gamma_{1}(r)\right)=0 \quad \text { ou } \quad \lim _{r \rightarrow 0}\left(\gamma_{2}(r)-\gamma_{0}(r)\right)=0
$$

De qualquer forma, temos uma contradição .

\subsection{Caso redutível}

Seja $(C, 0)$ um germe de curva analítica complexa em $\mathbb{C}^{2}$, com ramos $C=\cup_{i \in I} C_{i}$. Seja $m_{i}$ a multiplicidade de $\left(C_{i}, 0\right) ; i \in I$. Para cada par $i \neq j \in I$, por analogia com o caso de curvas reais, consideremos o seguinte número:

$$
\operatorname{sh}\left(C_{i}, C_{j}\right)=\operatorname{ord}_{r}\left(\operatorname{dist}\left(C_{i} \cap S_{r}(0), C_{j} \cap S_{r}(0)\right)\right)
$$

E, consideremos também,

$$
\operatorname{coinc} .\left(C_{i}, C_{j}\right)=\max \left\{\operatorname{ord}_{x}\left[y_{k}\left(x^{\frac{1}{m_{i}}}\right)-z_{l}\left(x^{\frac{1}{m_{j}}}\right)\right] \mid 1 \leqslant k \leqslant m_{i}, 1 \leqslant l \leqslant m_{j}\right\}
$$

em que $\left\{y_{k}\left(x^{\frac{1}{m_{i}}}\right)\right\}_{k=1}^{m_{i}}$ é o conjunto das parametrizações de Newton-Puiseux de $C_{i} \mathrm{e}$ $\left\{z_{l}\left(x^{\frac{1}{m_{j}}}\right)\right\}_{l=1}^{m_{j}}$ é o conjunto das parametrizações de Newton-Puiseux de $C_{j}$. O número coinc. $\left(C_{i}, C_{j}\right)$ é conhecido como coincidência entre $C_{i}$ e $C_{j}$ (cf.[21]).

\section{Lema 3.3.1.}

$$
\operatorname{sh}\left(C_{i}, C_{j}\right)=\text { coinc. }\left(C_{i}, C_{j}\right), \quad \forall \quad i \neq j \quad \text { em } \quad I .
$$


Prova. Sejam $\Gamma_{i}, \Gamma_{j}$ arcos de teste em $C_{i}$ e $C_{j}$, respectivamente, descritos por

$$
\Gamma_{k}(r)=\left(r, \tilde{y}_{k}\left(r^{\frac{1}{m_{k}}}\right)\right) \in \Gamma_{k} \cap S_{r}(0) ; k=i, j
$$

em que $\tilde{y}_{i}\left(x^{\frac{1}{m_{i}}}\right)$ e $\tilde{y}_{j}\left(x^{\frac{1}{m_{j}}}\right)$ são parametrizações de Newton-Puiseux de $C_{i}$ e $C_{j}$ tais que

$$
\operatorname{coinc} .\left(C_{i}, C_{j}\right)=\operatorname{ord}_{x}\left[\bar{y}_{i}\left(x^{\frac{i}{m_{i}}}\right)-\tilde{y}_{j}\left(x^{\frac{1}{m_{j}}}\right)\right]
$$

Então,

$$
\left.\operatorname{dist}\left(C_{i} \cap S_{r}(0), C_{j} \cap S_{r}(0)\right)\right) \leqslant\left\|\Gamma_{i}(r)-\Gamma_{j}(r)\right\|=\left|\tilde{y}_{i}\left(r^{\frac{1}{m_{i}}}\right)-\tilde{y}_{j}\left(r^{\frac{1}{m_{j}}}\right)\right| .
$$

Portanto, $\operatorname{sh}\left(C_{i}, C_{j}\right) \geqslant \operatorname{coinc} .\left(C_{i}, C_{j}\right)$.

Por outro lado, temos

$$
\tilde{y}_{k}\left(x^{\frac{1}{m_{k}}}\right)=h\left(x^{\frac{1}{m_{k}}}\right)+g_{k}\left(x^{\frac{1}{m_{k}}}\right), k=i, j
$$

$\operatorname{com} \operatorname{ord}_{x}\left[g_{i}\left(x^{\frac{1}{m_{i}}}\right)-g_{j}\left(x^{\frac{1}{m_{j}}}\right)\right]=\min \left\{\operatorname{ord}_{x} g_{i}\left(x^{\frac{1}{m_{i}}}\right), \operatorname{ord}_{x} g_{j}\left(x^{\frac{1}{m_{j}}}\right)\right\}$.

Sejam $\Gamma_{i} \subset C_{i}, \Gamma_{j} \subset C_{j}$ arcos de teste tais que

$$
\left.\left.\operatorname{dist}\left(C_{i} \cap S_{r}(0)\right), C_{j} \cap S_{r}(0)\right)=\operatorname{dist}\left(\Gamma_{i} \cap S_{r}(0)\right), \Gamma_{j} \cap S_{r}(0)\right) .
$$

Seja

$$
\Gamma_{k}(r)=\left(r e^{i \gamma_{k}(r)}, \tilde{y}_{k}\left(r^{\frac{1}{m_{k}}} e^{i \frac{\gamma_{k}(r)}{m_{k}}}\right)\right) \in S_{r}(0) \cap \Gamma_{k}, \quad k=i, j
$$

$\therefore$

$\operatorname{ord}_{r}|| \Gamma_{i}(r)-\Gamma_{j}(r) \|=\min \left\{1+\operatorname{ord}_{r}\left|e^{i \gamma_{i}(r)}-e^{i \gamma_{j}(r)}\right|, \operatorname{ord}_{r}\left|\tilde{y}_{i}\left(r^{\frac{1}{m_{i}}} e^{i \frac{\gamma_{i}(r)}{m_{i}}}\right)-\tilde{y}_{j}\left(r^{\frac{1}{m_{j}}} e^{i \frac{\gamma_{2}(r)}{m_{j}}}\right)\right|\right.$.

Mas, como

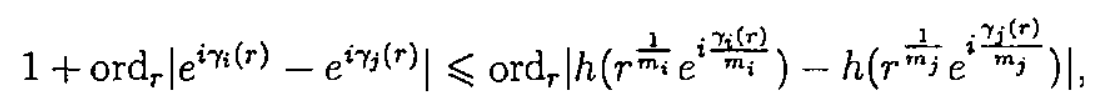

temos

$$
\operatorname{ord}_{r}\left\|\Gamma_{i}(r)-\Gamma_{j}(r)\right\| \leqslant \min \left\{\operatorname{ord}_{r} g_{i}\left(r^{\frac{1}{m_{i}}} e^{i \frac{\gamma_{i}(r)}{m_{i}}}\right), \operatorname{ord}_{r} g_{j}\left(r^{\frac{1}{m_{j}}} e^{\frac{\gamma_{j}(r)}{m_{j}}}\right)=\operatorname{coinc}\left(C_{i}, C_{j}\right) .\right.
$$

Isto é, $\operatorname{sh}\left(C_{i}, C_{j}\right) \leqslant$ coinc. $\left(C_{i}, C_{j}\right)$. 
Portanto, segue-se o resultado.

O teorema seguinte e o Corolário 3.4 .4 são, para a classificação bi-Lipschitz, os análogos ao teorema apresentado em [27] p. 888 para a classificação topológica.

Teorema 3.3.2. Dadas $(X, 0)$ e $(\tilde{X}, 0)$ curvas analíticas complexas em $\mathbb{C}^{2}$ com ramos $X=\cup_{i \in I} X_{i}$ e $\tilde{X}=\cup_{j \in J} \widetilde{X}_{j}$, as seguintes condições são equivalentes:

(i) Existe um germe de aplicação subanalítica bi-Lipschitz $F:(X, 0) \rightarrow(\widetilde{X}, 0)$;

(ii) Existe uma bijeção $\phi: I \rightarrow J$ tal que $\beta\left(X_{i}\right)=\beta\left(\tilde{X}_{\phi(i)}\right)$ e $\operatorname{sh}\left(X_{i}, X_{j}\right)=$ $\operatorname{sh}\left(\tilde{X}_{\phi(i)}, \tilde{X}_{\phi(j)}\right) ;$

(iii) Existe uma bijeção $\phi: I \rightarrow J$ tal que $\beta\left(X_{i}\right)=\beta\left(\widetilde{X}_{\phi(i)}\right) e\left(X_{i}, X_{j}\right)_{0}=\left(\widetilde{X}_{\phi(i)}, \widetilde{X}_{\phi(j)}\right)_{0}$;

(iv) $(X, 0)$ é topologicamente equivalente a $(\widetilde{X}, 0)$;

(v) existem um inteiro d, um germe de curva $(C, 0) \subset\left(\mathbb{C}^{d}, 0\right)$ e projeções lineares $p, \tilde{p}: \mathbb{C}^{d} \rightarrow \mathbb{C}^{2}$, ambas gerais para $C$ em 0 , tais que $p(C)=X$ e $\tilde{p}(C)=\tilde{X}$.

Prova. (i) $\Rightarrow($ ii $)$ Seja $F:(X, 0) \rightarrow(\tilde{X}, 0)$ um germe de aplicação subanalítica bi-Lipschitz. Por argumentos topológicos, podemos supor que $I=J$ e $F\left(X_{i}\right)=$ $\widetilde{X}_{i}, \forall i \in I$. Segue do Teorema 3.2.4 que $\beta\left(X_{i}\right)=\beta\left(\widetilde{X}_{i}\right), \forall i \in I$. Agora, consideremos $i \neq j \in I$ e mostremos que $\operatorname{sh}\left(X_{i}, X_{j}\right)=\operatorname{sh}\left(\widetilde{X}_{i}, \widetilde{X}_{j}\right)$. Para isso, tomemos $\Gamma_{1}, \Gamma_{2} \operatorname{arcos}$ de teste em $X$ tais que $\Gamma_{i} \subset X_{i}, i=1,2 \mathrm{e}$

$$
\operatorname{dist}\left(X_{i} \cap S_{r}(0), X_{j} \cap S_{r}(0)\right)=\operatorname{dist}\left(\Gamma_{i} \cap S_{r}(0), \Gamma_{j} \cap S_{r}(0)\right) .
$$

Observemos que $F\left(\Gamma_{1}\right), F\left(\Gamma_{2}\right)$ são arcos de teste em $\widetilde{X}$ tais que $\left.F\left(\Gamma_{i}\right) \subset \widetilde{(} X_{i}\right), i=1,2$. Daí, temos que

$$
\operatorname{dist}\left(\widetilde{X}_{i} \cap S_{r}(0), \widetilde{X}_{j} \cap S_{r}(0)\right) \leqslant \operatorname{dist}\left(F\left(\Gamma_{i}\right) \cap S_{r}(0), F\left(\Gamma_{j}\right) \cap S_{r}(0)\right) .
$$

Agora, como $F$ é bi-Lipschitz, podemos lançar mão do Teorema 2.1.3 para obter a relação

$$
\operatorname{dist}\left(\Gamma_{i} \cap S_{r}(0), \Gamma_{j} \cap S_{r}(0)\right) \approx \operatorname{dist}\left(F\left(\Gamma_{i}\right) \cap S_{r}(0), F\left(\Gamma_{j}\right) \cap S_{r}(0)\right) .
$$


E, portanto, $\operatorname{sh}\left(X_{i}, X_{j}\right) \geqslant \operatorname{sh}\left(\tilde{X}_{i}, \tilde{X}_{j}\right)$.

Com um argumento completamente simétrico ao usado acima, provamos que $\operatorname{sh}\left(X_{i}, X_{j}\right) \leqslant \operatorname{sh}\left(\widetilde{X}_{i}, \tilde{X}_{j}\right)$. E, assim, concluímos a igualdade desses contatos.

(ii) $\Rightarrow$ (iii) Suponhamos que $I=J, \beta\left(X_{i}\right)=\beta\left(\tilde{X}_{i}\right), \forall, i \in I$ e $\operatorname{sh}\left(X_{i}, X_{j}\right)=$ $\operatorname{sh}\left(\widetilde{X}_{i}, \tilde{X}_{j}\right), \forall i \neq j \in I$. Assim, pelo Lema 3.3.1, temos que coinc. $\left(X_{i}, X_{j}\right)=$ coinc. $\left(\widetilde{X}_{i}, \widetilde{X}_{j}\right), \forall i \neq j \in I$. Em [21], M. Merle obtém uma releitura do índice de interseção dos ramos de uma curva a partir da coincidência desses ramos. Fazendo uso dessa relação, temos $\left(X_{i}, X_{j}\right)_{0}=\left(\widetilde{X}_{i}, \widetilde{X}_{j},\right)_{0}, \forall i \neq j \in I$.

$($ iii) $\Rightarrow($ iv $)$ Veja Teorema 3.0.11. (iv) $\Rightarrow(v)$ Veja [27] p. 888.

$(v) \Rightarrow(i)$ Sejam $d$, um inteiro, $(C, 0) \subset\left(\mathbb{C}^{d}, 0\right)$ um germe de curva e $p, \tilde{p}: \mathbb{C}^{d} \rightarrow \mathbb{C}^{2}$ projeçōes lineares, ambas gerais para $C$ em 0 , tais que $p(C)=X$ e $\tilde{p}(C)=\widetilde{X}$. Então, $\tilde{p} \circ p^{-1}:(X, 0) \rightarrow(\widetilde{X}, 0)$ é o germe de uma aplicação subanalítica bi-Lipschitz.

\subsection{Tipo bi-Lipschitz de curvas complexas.}

Os resultados obtidos na seção anterior sugerem, naturalmente, as seguintes perguntas:

(i) Dados dois germes de curvas complexas espaciais, eles são topologicamente equivalentes se, e somente se, existe um germe de aplicação subanalítica biLipschitz entre eles?

(ii) O semigrupo de uma curva espacial irredutível é um invariante métrico?

Com o objetivo de responder às perguntas acima, consideremos os seguintes germes de curvas analíticas complexas espaciais pela origem de $\mathbb{C}^{3}$.

$$
\begin{aligned}
& X_{1}: x=t^{6}, \quad y=t^{14}, \quad z=t^{17} \\
& X_{2}: \quad x=t^{6}, \quad y=t^{14}, \quad z=t^{39} \\
& X_{3}: \quad x=t^{6}, \quad y=t^{14}+t^{17}, \quad z=t^{39} .
\end{aligned}
$$


Denotemos por $\Gamma\left(X_{i}\right)$ o semigrupo da curva $\left(X_{i}, 0\right), i=1,2,3$. Uma projeção genérica de $\left(X_{1}, 0\right)$ no plano tem $(6,14,17)$ como seqüência de expoentes característicos e uma projeção genérica de $\left(X_{3}, 0\right)$ no plano tem $(6,14,17)$ como seqüência de expoentes característicos. Pelo Teorema 3.3.2, existe um germe de aplicação subanalítica bi-Lipschitz $F:\left(X_{1}, 0\right) \rightarrow\left(X_{3}, 0\right)$, enquanto $\Gamma\left(X_{1}\right)=\langle 6,14,17\rangle \neq$ $\langle 6,14,39\rangle=\Gamma\left(X_{3}\right)$. Quanto a $X_{2}$, temos que uma projeção genérica de $\left(X_{2}, 0\right)$ no plano tem $(6,14,39)$ como seqüência de expoentes característicos, logo não existe germe de aplicação subanalítica bi-Lipschitz $F:\left(X_{2}, 0\right) \rightarrow\left(X_{3}, 0\right)$, por outro lado, $\Gamma\left(X_{2}\right)=\langle 6,14,39\rangle=\Gamma\left(X_{3}\right)$. Estes exemplos respondem negativamente à Pergunta (ii). De fato, vimos que o semigrupo de uma curva analítica complexa espacial não tem qualquer influência no seu comportamento métrico. Quanto à Pergunta (i), vimos que a equivalência topológica de dois germes de curvas complexas espaciais não acarreta na existência de um germe de aplicação subanalítica bi-Lipschitz entre eles, pois $\left(X_{2}, 0\right)$ é topologicamente equivalente a $\left(X_{3}, 0\right)$ (cf. Teorema $2.1[14]$ ). Ainda a respeito da Pergunta (i), podemos afirmar o seguinte: a existência de um germe de aplicação subanalítica bi-Lipschitz entre dois germes de curvas espaciais implica na equivalência topológica desses germes (ver Corolário 3.4.2).

Lema 3.4.1. Sejam $(X, 0)$ um germe de curva analítica complexa em $\mathbb{C}^{n}, n \geqslant 3$, e $p$ : $\mathbb{C}^{n} \rightarrow \mathbb{C}^{2}$ uma projeção linear geral para $X$ em 0 . Consideremos uma decomposição $\mathbb{C}^{n}=\mathbb{C}^{2} \times \mathbb{C}^{n-2}$ e coordenadas $(x, y)$ tais que $p(x, y)=x$. Entäo, existe um germe de aplicação subanalítica bi-Lipschitz $\Phi:\left(\mathbb{C}^{n}, 0\right) \rightarrow\left(\mathbb{C}^{n}, 0\right)$ tal que $\Phi(X)=p(X) \times\{0\}$. Em particular, $(X, 0)$ é topologicamente equivalente a $(p(X) \times\{0\}, 0)$ como germes de curvas em $\mathbb{C}^{n}$.

Prova. Claramente, é suficiente provarmos para o caso $n=3$. Como $p$ é geral para $X$ em $0, X$ é o gráfico de uma função subanalítica Lipschitz $f: p(X) \subset \mathbb{C}^{2} \rightarrow \mathbb{C}$. Pelo Teorema de Kirszbraun, seja $F: \mathbb{C}^{2} \rightarrow \mathbb{C}$ uma extensāo subanalítica Lipschitz de $f$. Entāo, $\Phi:\left(\mathbb{C}^{3}, 0\right) \rightarrow\left(\mathbb{C}^{3}, 0\right)$ definida por $\Phi(x, y)=(x, y-F(x))$ é um germe de homeomorfismo subanalítico bi-Lipschitz tal que $\Phi(X)=p(X) \times\{0\}$. 
Corolário 3.4.2. Sejam $(X, 0)$ e $(Y, 0)$ germes de curvas analíticas complexas em $\mathbb{C}^{n}$. Se existe um germe de aplicação subanalítica bi-Lipschitz $F:(X, 0) \rightarrow(Y, 0)$, então $(X, 0)$ é topologicamente equivalente a $(Y, 0)$ em $\mathbb{C}^{n}$.

Observação 3.4.3. De fato, provaremos que vale um resultado bem mais forte do que o Corolário 3.4.2. A saber, provaremos que se existe um germe de aplicação subanalítica bi-Lipschitz $F:(X, 0) \rightarrow(Y, 0)$, então existe um germe de aplicação bi-Lipschitz $\Phi:\left(\mathbb{C}^{n}, 0\right) \rightarrow\left(\mathbb{C}^{n}, 0\right)$ tal que $\Phi(X)=Y$.

A construção seguinte tem como objetivo demonstrar o que afirmamos na observação acima. Por analogia ao raciocínio empregado no capítulo sobre curvas reais para resolver o problema de classificação do tipo bi-Lipschitz, consideramos primeiro o caso plano, isto é, $n=2$.

Sejam $(X, 0)$ e $(\tilde{X}, 0)$ germes de curvas analíticas complexas em $\mathbb{C}^{2}$. Sejam $d$, um inteiro, $(C, 0) \subset\left(\mathbb{C}^{d}, 0\right)$ um germe de curva e $p, \tilde{p}: \mathbb{C}^{d} \rightarrow \mathbb{C}^{2}$ projeçōes lineares, ambas gerais para $C$ em 0 , tais que $p(C)=X$ e $\tilde{p}(C)=\widetilde{X}$. Como o conjunto das projeções gerais para $C$ em 0 , é um aberto de Zariski no espaço das projeções lineares $\mathbb{C}^{d} \rightarrow \mathbb{C}^{2}$, temos um caminho $p_{t}$ nesse espaço formado por projeções lineares gerais para $C \mathrm{em}$ 0 satisfazendo $p_{0}=p$ e $p_{1}=\tilde{p}$. Seja $\Omega=\left\{(x, t) \in \mathbb{C}^{2} \times I: x \in X_{t}=p_{t}(C)\right\}$. Temos $\phi_{t}:=p_{t} \circ p^{-1}: X, 0 \rightarrow X_{t}, 0$ bi-Lipschitz para cada $t \in I=[0,1]$. Como $I$ é compacto, podemos escolher constantes de Lipschitz para $\phi_{t}$ independentes de $t$. Então,

$$
V(x, t):=\left(\frac{\partial \phi_{t}}{\partial t}(x, t), t\right)
$$

define um campo de vetores Lipschitz sobre $\Omega$. Pelo Teorema de Kirszbraun, $V$ se estende a um campo Lipschitz sobre $\mathbb{C}^{2} \times I$ o qual é tangente a $\Omega$. Portanto, tomando o fluxo de $V$, dado pela condição $(x, t, 0) \mapsto(x, t)$, no tempo $t=1$ obtemos um germe de aplicação bi-Lipschitz $F:\left(\mathbb{C}^{2}, 0\right) \rightarrow\left(\mathbb{C}^{2}, 0\right)$ tal que $F(X)=\tilde{X}$.

Corolário 3.4.4. Dadas $(X, 0)$ e $(\widetilde{X}, 0)$ curvas analíticas complexas em $\mathbb{C}^{2}$ com ramos $X=\cup_{i \in I} X_{i}$ e $\tilde{X}=\cup_{j \in J} \tilde{X}_{j}$, as seguintes condiçöes são equivalentes:

(i) Existe um germe de aplicação bi-Lipschitz $F:\left(\mathbb{C}^{2}, 0\right) \rightarrow\left(\mathbb{C}^{2}, 0\right)$ tal que $F(X)=$ $\widetilde{X}$ 
(ii) Existe um germe de aplicação subanalítica bi-Lipschitz $F:(X, 0) \rightarrow(\widetilde{X}, 0)$;

(iii) Existe uma bijeção $\phi: I \rightarrow J$ tal que $\beta\left(X_{i}\right)=\beta\left(\tilde{X}_{\phi(i)}\right)$ e $\operatorname{sh}\left(X_{i}, X_{j}\right)=$ $\operatorname{sh}\left(\widetilde{X}_{\phi(i)}, \widetilde{X}_{\phi(j)}\right)$

(iv) Existe uma bijeção $\phi: I \rightarrow J$ tal que $\beta\left(X_{i}\right)=\beta\left(\tilde{X}_{\phi(i)}\right)$ e $\left(X_{i}, X_{j}\right)_{0}=\left(\widetilde{X}_{\phi(i)}, \widetilde{X}_{\phi(j)}\right)_{0}$;

(v) $(X, 0)$ é topologicamente equivalente a $(\widetilde{X}, 0)$;

(vi) existem um inteiro d, um germe de curva $(C, 0) \subset\left(\mathbb{C}^{d}, 0\right)$ e projeções lineares $p, \tilde{p}: \mathbb{C}^{d} \rightarrow \mathbb{C}^{2}$, ambas gerais para $C$ em 0 , tais que $p(C)=X$ e $\tilde{p}(C)=\tilde{X}$.

Corolário 3.4.5. Sejam $(X, 0) e(\widetilde{X}, 0)$ germes de curvas analíticas complexas em $\mathbb{C}^{n}$. Se existe um germe de aplicação subanalítica bi-Lipschitz $F:(X, 0) \rightarrow(\widetilde{X}, 0)$, então existe um germe de aplicação bi-Lipschitz $F:\left(\mathbb{C}^{n}, 0\right) \rightarrow\left(\mathbb{C}^{n}, 0\right)$ tal que $F(\mathcal{X})=\tilde{X}$.

Prova. Idêntica à prova dada para o Corolário 3.4.2.

O seguinte corolário, segue imediatamente do resultado, devido a Lê-Ramanujam, o qual estabelece que o número de Milnor é um invariante completo para garantir a trivialidade topológica de famílias de hipersuperfícies complexas em $\mathbb{C}^{n} ; n \neq 3$.

Corolário 3.4.6. Seja $X_{t}$ uma família de germes de curvas complexas planas ao longo de um intervalo compacto $I$, com equação dada por $f_{t}(x, y)=0$. Se o número de Milnor $\mu\left(f_{t}\right)$ independe de $t$, então a familia $\left\{\left(X_{t}, 0\right)\right\}$ é bi-Lipschitz trivial ao longo de $I$. 


\section{Capítulo 4}

\section{bi-Lipschitz trivialidade de funções}

Nos capítulos anteriores, tratamos do problema de classificação de conjuntos singulares. Uma outra questão, seria o problema de classificação de aplicações. Para o problema de classificação topológica, o seguinte resultado devido a H.C. King [17], mostra que as duas questões acima não estão distantes uma da outra.

Dizemos que uma família de germes de aplicações $f_{z}:\left(\mathbb{R}^{n}, 0\right) \rightarrow\left(\mathbb{R}^{k}, 0\right) z \in \mathbb{R}^{p}$ não possui fusão de pontos críticos se existe um aberto $U$ de $\mathbb{R}^{n}$ contendo a origem tal que, para cada $z, f_{z}: U \rightarrow \mathbb{R}^{k}$ é uma singularidade isolada na origem.

Teorema 4.0.7. Seja $f_{z}:\left(\mathbb{R}^{n}, 0\right) \rightarrow\left(\mathbb{R}^{k}, 0\right) z \in \mathbb{R}^{p}$ uma familia de germes "sem fusão de pontos críticos"e suponha que exista uma familia de germes de homeomorfismos $g_{z}$ tais que $g_{z}\left(f_{z}^{-1}(0)\right)=f_{0}^{-1}(0)$. Então, existe uma familia de germes de homeomorfismos $h_{z}:\left(\mathbb{R}^{n}, 0\right) \rightarrow\left(\mathbb{R}^{n}, 0\right) z \in \mathbb{R}^{p}$ e uma vizinhança $V$ de 0 em $\mathbb{R}^{p}$ tais que $f_{z} \circ h_{z}=f_{0}, \forall z \in V$.

A classificação bi-analítica dos germes de aplicaçōes analíticas, como já vimos em outra ocasião, com seguinte exemplo de H. Whitney é um projeto difícil.

$$
W(x, y, t)=x y(x-y)(x-t y) \quad t>1
$$

Relembro que o exemplo acima motiva vários autores a considerar relações de equivalência, nesse caso sobre todos os germes de aplicaçōes analíticas, as quais são mais fortes do que homeomorfismos topológicos e mais fracas do que homeomorfismos 
bi-analíticos. Em [19], por exemplo,T-C. Kuo introduz o conceito de trivialização analítica modificada (MAT) a qual induz uma relação de equivalência no conjunto de todos os germes de funções analíticas reais satisfazendo a propriedade de ser mais forte do que topológica e mais fraca do que bi-analítica. Ainda em [19], T-C. Kuo mostra o seguinte

Teorema 4.0.8. Seja $F(x, t)=H_{k}(x, t)+H_{k+1}(x, t)+\cdots ; \quad H_{k}(x, t) \neq 0$, em que $H_{i}(x, t)$ é uma forma homogênea de grau $i$ em $x$. Se $H_{k}(x, t)$ não é degenerada em $x$, para $t \in I$, então $F(x, t)$ admite uma MAT ao longo do intervalo $I$.

Aqui, exploramos o seguinte conceito métrico: seja $F:\left(\mathbb{R}^{n} \times \mathbb{R},\{0\} \times \mathbb{R}\right) \rightarrow\left(\mathbb{R}^{p}, 0\right)$ uma família de germes de aplicações pela origem de $\mathbb{R}^{n}$. Dizemos que $F$ admite uma bi-Lipschitz (ou $\mathcal{R}$-bi-Lipschitz) trivialização ao longo de um intervalo $I$ se existem $t_{0} \in I$ e um germe de homeomorfismo bi-Lipschitz $\phi:\left(\mathbb{R}^{n} \times I,\{0\} \times I\right) \rightarrow\left(\mathbb{R}^{n} \times I,\{0\} \times I\right)$ preservando níveis tal que $F(\phi(x, t))=$ $F\left(x, t_{0}\right)$.

Abaixo, apresentamos um exemplo bem didático que deixa clara a diferença entre a trivializaçāo bi-Lipschitz da família dos zeros de uma família de aplicações e a trivializaçāo bi-Lipschitz dessa família de aplicaçōes.

Exemplo 4.0.9. Seja $f_{t}: \mathbb{R}, 0 \rightarrow \mathbb{R}$ definida por $f_{t}(x)=x^{3}+t x$ com $t \in I=[0,1]$. Claramente, $f_{t}$ não é bi-Lipschitz trivial ao longo de I. Por outro lado, $f_{t}^{-1}(0)=0$ para cada $t \in I$.

O exemplo acima mostra que nāo é possível obtermos um resultado do "tipo King" para a categoria bi-Lipschitz.

Exemplo 4.0.10. (J. Briançon, J:P. Speder [7]) Seja $f_{t}:\left(\mathbb{R}^{3}, 0\right) \rightarrow\left(\mathbb{R}^{3}, 0\right)$ a família definida por $f_{t}(x, y, z)=z^{5}+x y^{7}+x^{15}+t y^{6} z$. Em [13] os autores provam que $f_{t}$ admite uma trivialização analítica modificada ao longo de qualquer intervalo que não contenha $t_{0}=-15^{\frac{1}{7}}\left(\frac{7}{2}\right)^{\frac{4}{3}} \frac{1}{3}$. Por outro lado, é bem conhecido que $f_{t}$ não satisfaz as condições de regularidade de Whitney, portanto, não admite uma trivialização bi-Lipschitz, obtida por integraçāo de um campo de vetores Lipschitz, ao longo de 
qualquer intervalo contendo $t=0$. Este fato é suficiente para garantirmos que o seguinte teorema não decorre do teorema acima de T-C. Kuo.

Teorema 4.0.11. Seja $F(x, t)=H_{k}(x, t)+H_{k+1}(x, t)+\cdots ; \quad H_{k}(x, t) \neq 0$, em que $H_{i}(x, t)$ é uma forma homogênea de grau $i$ em $x$. Se $H_{k}(x, t)$ não é degenerada em $x$, para $t \in I$, então $F(x, t)$ admite uma bi-Lipschitz trivialização ao longo do intervalo $I$.

Nesta seção, apresentamos resultados sobre a bi-Lipschitz determinação de germes de polinômios quase-homogêneos e obtemos o teorema acima como caso particulardesses resultados. As idéias que utilizamos nesta seçāo estão completamente contidas no artigo [25], donde podemos afirmar que os resultados seguintes vêm como exercícios diante de [25]. Contudo, optei por tê-los enunciados em minha tese, pois considero a teoria da $\mathcal{R}$-equivalência bi-Lipschitz carente de referências.

\subsection{Um resultado do tipo Kuo}

Seja $C(n, p)$ o espaço de germes de aplicações suaves $\mathbb{R}^{n}, 0 \rightarrow \mathbb{R}^{p}$. Dada $f \in C(n, p)$ denotemos por $I_{\mathcal{R}} f$ o ideal de $C_{n}:=C(n, 1)$ gerado pelos $p \times p$ menores da matriz. jacobiana de $f$ e seja $N_{\mathcal{R}} f(x)=\sum_{j} M_{j}^{2}$, em que os $M_{j}$ são os geradores de $I_{\mathcal{R}} f$. Dizemos que $N_{\mathcal{R}} f(x)$ satisfaz uma condição de Lojasiewicz se existem constantes $c>0$ e $\alpha>0$ tais que $N_{\mathcal{R}} f(x) \geqslant c\|x\|^{\alpha}$.

Definição 4.1.1. Dada $\left(r_{1}, \ldots, r_{n}\right)$ uma $n$-upla de inteiros positivos, para qualquer monômio $x^{\alpha}=x_{1}^{\alpha_{1}} \cdots x_{n}^{\alpha_{n}}$, definimos $\operatorname{fil}\left(x^{\alpha}\right)=\sum_{i=1}^{n} \alpha_{i} r_{i}$.

Definimos uma filtração no anel $C_{n}$ via a função definida por fil $(f)=\min \left\{\operatorname{fil}\left(x^{\alpha}\right)\right.$ : $\left.\left(\frac{\partial^{f}}{\partial x^{\alpha}}\right)(0) \neq 0\right\}$, para qualquer germe $f$ em $C_{n}$. Esta definição pode ser estendida para $C_{n+r}$ o anel de famílias a $r$-parâmetros de germes de funções de $n$-variáveis, definindo $\operatorname{fil}\left(x^{\alpha} t^{\beta}\right)=\operatorname{fil}\left(x^{\alpha}\right)$.

Para qualquer germe de aplicação $f=\left(f_{1}, \ldots, f_{p}\right) \in C(n, p)$, definimos fil $(f)=$ $\left(d_{1}, \ldots, d_{p}\right)$, em que $d_{i}=\operatorname{fil}\left(f_{i}\right)$, para cada $i=1, \ldots p$. E denotamos, $\min \operatorname{fil}(f)=$ $\min \left\{d_{1}, \ldots, d_{p}\right\}$. 
Fixemos $\left(r_{1}, \ldots, r_{n} ; 2 k\right)$. Definimos a função controle padrão $\rho(x)$ por $\rho(x)=$ $x^{2 \alpha_{1}}+\cdots+x^{2 \alpha_{n}}$, em que os $\alpha_{i}$ são escolhidos de sorte que a função $\rho$ seja quasehomogênea de tipo $\left(r_{1}, \ldots, r_{n} ; 2 k\right)$.

Relembramos que associada aos pesos $\left(r_{1}, \ldots, r_{n}\right)$ temos a seguinte ação sobre $\mathbb{R}^{n}$ :

$$
\lambda \cdot p=\left(\lambda^{r_{1}} p_{1}, \ldots, \lambda^{r_{n}} p_{n}\right) .
$$

Lema 4.1.2. Sejam $h(x)$ um polinômio quase-homogêneo de tipo $\left(r_{1}, \ldots, r_{n} ; 2 k\right)$, com $r_{1} \leqslant \cdots \leqslant r_{n}$, $\rho$ a função controle padrão de mesmo tipo que $h$ e $h_{t}(x)$ uma familia de germes de funções tal que

$$
\text { fil }\left(h_{t}\right) \geqslant 2 k+r_{n}, \quad t \in[0,1] .
$$

Então, a função $\frac{h_{t}(x)}{\rho(x)}$ é Lipschitz.

Prova. Sem perda de generalidade, podemos supor $h_{t}(x)$ quase-homogênea de tipo $\left\langle r_{1}, \ldots, r_{n} ; d\right)$ com $d \geqslant 2 k+r_{n}$. Consideremos $\left.G_{t}(x, y)=\mid \rho(y) h_{t}(x)-\rho(x) h_{t}(y)\right\}$, $m_{t}(x, y)=\|x-y\| \rho(x) \rho(y)$ e $M=\left\{(x, y, t): G_{t}(x, y)=1\right\}$. Como $M$ é fechado, o número $\left.c=\inf \left\{m_{t}(x, y):(x, y, t) \in M\right)\right\}$ é positivo. Agora, sejam $x, y \in \mathbb{R}^{n}$, suficientemente próximos da origem, tais que $x \neq 0, y \neq 0$ e $x \neq y$. Seja $\lambda>0$ tal que $G_{t}(\lambda \cdot x, \lambda \cdot y)=1$, isto é,

$$
G_{t}(x, y)=\frac{1}{\lambda^{2 k+d}}
$$

Por outro lado, usamos que $\lambda>1$ e obtemos:

$$
\begin{aligned}
m_{t}(\lambda \cdot x, \lambda \cdot y) & =\lambda^{4 k}\|\lambda \cdot x-\lambda \cdot y\| \rho(x) \rho(y) \\
& \leqslant \lambda^{4 k+r_{n}}\|x-y\| \rho(x) \rho(y) \\
& =\lambda^{4 k+r_{n}} m_{t}(x, y) \\
m_{t}(x, y) & \geqslant \frac{1}{\lambda^{4 k+r_{n}}} c .
\end{aligned}
$$

$\therefore$

Agora, como $\lambda>1$ e $d \geqslant 2 k+r_{n}$, segue de (4.2) e (4.3) que $m_{t}(x, y) \geqslant c G_{t}(x, y)$, ou seja,

$$
\left\|\frac{h_{t}(x)}{\rho(x)}-\frac{h_{t}(y)}{\rho(y)}\right\| \leqslant c^{-1}\|x-y\|
$$


Como conseqüência do lema acima temos a seguinte

Proposição 4.1.3. Seja $f_{t}(x)=f(x)+t \Theta(x, t), t \in[0,1]$, uma deformação de um germe de função polinomial quase-homogênea $f$ de tipo $\left(r_{1}, \ldots, r_{n} ; d\right), \operatorname{com} r_{1} \leqslant \cdots \leqslant$ $r_{n}$ e satisfazendo uma condição de Lojasiewicz $N_{\mathcal{R}} f_{t}(x) \geqslant\|x\|^{\alpha}$, para constantes c e $\alpha$. Se fil $(\Theta) \geqslant d+r_{n}-r_{1}$, então $f_{t}$ admite uma bi-Lipschitz trivialização ao longo de $I=[0,1]$.

Prova. Observamos que para cada $i$ existe um $s_{i}$ tal que $\frac{\partial f}{\partial x_{i}}$ é quase-homogêneo de tipo $\left(r_{1}, \ldots, r_{n} ; s_{i}\right)$.

Seja $N_{\mathcal{R}}^{*} f_{i}$ definido por

$$
N_{\mathcal{R}}^{*} f=\sum\left[\frac{\partial f}{\partial x_{i}}\right]^{2 \alpha_{i}},
$$

$\operatorname{com} \alpha_{i}=\frac{k}{s_{i}}$ e $k=m . m . c .\left(s_{i}\right)$. Então, $N_{\mathcal{R}}^{*} f$ é uma função controle quase-homogênea de tipo $\left(r_{1}, \ldots, r_{n} ; 2 k\right)$.

O lema seguinte está provado em [25].

Lema 4.1.4. Existem constantes $0<c_{2}<c_{1}$ tais que

$$
c_{2} \rho(x) \leqslant N_{\mathcal{R}}^{*} f_{t} \leqslant c_{1} \rho(x)
$$

Temos a seguinte igualdade;

$$
\frac{\partial f_{t}}{\partial t} N_{\mathcal{R}}^{*} f_{t}=d f_{t}(W)
$$

em que $W$ é dado por

$$
W=\sum \frac{\partial f_{t}}{\partial t}\left[\frac{\partial f}{\partial x_{i}}\right]^{2 \alpha_{i}-1} \frac{\partial}{\partial x_{i}} .
$$

Como fil $\left(\frac{\partial f_{t}}{\partial t}\right) \geqslant d+r_{n}-r_{1} \mathrm{e}$

$$
\text { fil } \begin{aligned}
\left(\left[\frac{\partial f_{t}}{\partial x_{i}}\right]^{2 \alpha_{i}-1}\right) & =\left(2 \alpha_{i}-1\right) \text { fil }\left(\frac{\partial f_{t}}{\partial x_{i}}\right) \\
& =\left(2 \alpha_{i}-1\right)\left(d-r_{i}\right) \\
& =2 K-d+r_{i} \\
& \geqslant 2 k-d+r_{1}
\end{aligned}
$$


e, $\min \operatorname{fil}(W) \geqslant \operatorname{fil}(\Theta)+2 k-d+r_{1} \geqslant 2 k+r_{n}$

Seja $v: \mathbb{R}^{n} \times \mathbb{R}, 0 \rightarrow \mathbb{R}^{n} \times \mathbb{R}, 0$ o campo de vetores definido por $\frac{W}{N_{\mathcal{R}}^{*} f_{t}}$. Pelo Lema 4.1 .2 , temos que $v$ é um campo Lipschitz.

A equaçāo $\left(\frac{\partial f_{t}}{\partial t}\right)(x, t)=\left(d f_{t}\right)_{x}(x, t)(v(x, t))$ implica a bi-Lipschitz trivialidade da família $f_{t}(x)$ ao longo de um pequeno aberto em volta de $t=0$. Como o mesmo argumento é verdadeiro para todo $t=t_{0} \in I$, a prova está completa.

Observação 4.1.5. A respeito da proposição acima, se estamos no caşo quasehomogêneo puro, isto é, se $r_{n}>r_{1}$, então é suficiente pedir que $N_{\mathcal{R}}^{*} f$ satisfaça uma condição de Lojasiewicz para obter $N_{\mathcal{R}}^{*} f_{t}$ satisfazendo uma condição de Lojasiewicz.

Observação 4.1.6. Como conseqüência imediata da Proposição 4.1.3, obtemos o Teorema 4.0.11 e, portanto, também vale o:

Corolário 4.1.7. A familia (4.1) é bi-Lipschitz trivial ao longo de qualquer intervalo $I=[a, b] \operatorname{com} a>1$.

Em [30], D. Trotman prova que a multiplicidade de funções analíticas complexas é um invariante para a bi-Lipschitz equivalência. Em outro artigo menos recente, a saber [29], D. Trotman havia provado que a multiplicidade é invariante 'por difeomorfismos de classe $C^{1}$. Para justificar [30], D. Trotman comenta, de forma muito natural, que não é esperado que se duas funções $f$ e $g$ são bi-Lipschitz equivalentes então $f^{-1}(0)$ e $g^{-1}(0)$ são necessariamente $C^{1}$-equivalentes. De fato, o comentário dele estava correto e o corolário acima justifica essa afirmação.

A Proposição 4.1.3 ainda vale para aplicações com singularidade isolada na origem. Embora tais aplicações ocorram raramente, não me custa enunciar o seguinte

Proposição 4.1.8. Seja $f_{t}(x)=f(x)+t \Theta(x, t)$ uma deformação de um germe de aplicaçāo polinomial quase-homogênea $f \in C(n, p)$ de tipo $\left(r_{1}, \ldots, r_{n} ; d_{1}, \ldots, d_{p}\right)$, com $r_{1} \leqslant \cdots \leqslant r_{n}$ e com $d_{1} \leqslant \cdots \leqslant d_{p}$, satisfazendo uma condiçāo de Lojasiewicz $N_{\mathcal{R}} f_{t}(x) \geqslant c\|x\|^{\alpha}$, para constantes c e $\alpha$. Se fil $\left(\Theta_{i}\right) \geqslant d_{i}+r_{n}-r_{1}$, para todo $i e$ $t \in[0,1]$, então $f_{t}$ admite uma bi-Lipschitz trivialização ao longo de $I=[0,1]$. 
Substituíndo o Lema 2 de [25], pelo Lema 4.1.2, na demonstração da Proposição 2.2 de [25], obtemos uma prova para a Proposição 4.1.8.

Com esses resultados em mãos, uma pergunta natural a se formular é: quão boa é a estimativa apresentada na Proposição 4.1.8?

Vimos no Teorema 2.4.6 que toda família quase-homogênea de curvas planas é bi-Lipschitz trivial. Seria, então, interessante saber a respeito da $\mathcal{R}$-bi-Lipschitz trivialidade de famílias quase-homogêneas de funções de duas variáveis. Lembro que, essa pergunta não se põe no caso de mais de duas variáveis pois temos o exemplo de Briançon-Speder. O entendimento deste problema poderia trazer esclarecimentos para a questão da ocorrência ou não da modalidade em se tratando da $\mathcal{R}$-bi-Lipschitz equivalência. 


\section{Referências Bibliográficas}

[1] K. Bekka, (c)-regularité et trivialité topoligique, Singularity Theory and its Applications. Warwick 1989, part I. Lectures Notes in Mathematics no 1462, p.4262. Springer-Verlag.

[2] L. Birbrair , Local bi-Lipschitz classification of 2-dimensional semialgebraic sets. Houston J. Math. 25 (1999), no. 3, 453-472.

[3] L. Birbrair, A. Fernandes, Metric theory of semialgebraic curves, Rev. Mat. Complut. 13 (2000), no. 2, 369-382

[4] L. Birbrair, T. Mostowski, Normal embeddings of semialgebraic sets, Michigan Math. J. 47 (2000), no. 1, 125-132.

[5] R. Benedetti, J-J. Risler, Real algebraic and semi-algebraic sets, Actualités Mathématiques, Hermann, Paris (1990).

[6] J. Bochnak, J-J. Risler, Sur les exposants de Lojasiewicz, Comment. Math. Helv. 50 (1975), no. 4, 493-507.

[7] J. Briançon, J. Speder, La trivialité topologique n'implique pas les conditions de Whitney, C. R. Acad. Sci., 280 (1975), Paris, 365-367.

[8] E. Brieskorn, H. Knörrer, Plane algebraic curves, Birkhäuser Verlag (1986).

[9] J. Damon, Topological invariants $\mu$-constant deformations of complete intersection singularities. Oxford Quart. J. 158 (1989), 139-160. 
[10] Federer, Geometric measure theory, Springer-Verlag New York Inc., New York 1969.

[11] A.C.G. Fernandes, On metric theory of complex curves, submetido para publicação (2001).

[12] T. Fukui, S. Koike, T-C. Kuo, Blow-analytic equisingularities, properties, problems and progress, Real analytic and algebraic singularities, Pitman Res. Notes Math. Ser., 381, Longman, Harlow, 1998.

[13] T. Fukui, L. Paunescu, Modified analytic trivialization for weighted homogeneous function-germs, J. Math Soc. Japan, 52 (2000), no. 2, 433-446.

[14] T. Gaffney, $\mathcal{L}^{0}$-equivalence of maps, Math Proc. Phil. Soc. 128 (2000), 479-496.

[15] E. Garcia Barroso, Un théorème de décomposition pour. les polaires génériques d'une courbe plane.C. R. Acad. Sci. Paris Sér. I Math. 326 (1998), no. 1, 59-62

[16] C. Gibson, K. Wirthmüler, du Plessis A., Looijenga E. Topological stability of smooth mappings Lectures Notes in Mathematics, vol.552, Springer, 1976.

[17] H.C. King, Topological type in families of germs, Inventiones Math. 62 (1980) 1-13.

[18] S. Koike, On strong $C^{0}$-equivalence of real analytic functions, J. Math. Soc. Japan 45 (1993), no. 2, 313-320.

[19] T.C. Kuo, Modified analytic trivialization of singularities, J. Math. Soc. Japan $32(1980), 605-614$.

[20] E. McShane, Extensions of range of functions, Bull. Amer. Math. Soc. 40 (1934), 873-842.

[21] M. Merle, Invariants polaires des courbes planes, Invent. Math. 41 (1977), no. 2, 103-111.

[22] T. Mostowski, Lipschitz equisingularity, Dissertationes Math. 243 (1985). 
[23] T. Mostowski, Lipschitz stratification and Lipschitz isotopies, preprint 2001.

[24] A. Parusinski, Lipschitz stratification of subanalytic sets, Ann. Sci. École Norm. Sup. (4) 27 (1994), no. 6, 661-696.

[25] M.A.S. Ruas, M.J. Saia, $C^{l}$-determinacy of weighted homogeneous germs, Hokkaido Mathematical Journal, vol. 26, no 1 (1997), 89-99.

[26] R.N.A. dos Santos, Tese de doutorado, em preparação, ICMC-USP.

[27] B. Teissier, Introduction to curve singularities, Singularity theory (Trieste, 1991), 866-893, World Sci. Publishing, River Edge, NJ, 1995.

[28] B. Teissier, IVariétés polaires. II. Multiplicits polaires, sections planes, et conditions de Whitney, Algebraic geometry (La Rbida, 1981), 314-491, Lecture Notes in Math., 961, Springer, Berlin, 1982.

[29] D. Trotman, Bi-Lipschitz invariance of the multiplicity, Bull. London Math. Soc. 29 (1997), no. 2, 200-204.

[30] D. Trotman, Multiplicity as a $C^{1}$ invariant, Real analytic and algebraic singularities, Pitman Res. Notes Math. Ser., 381, Longman, Harlow, 1998.

[31] C.T.C. Wall, Finite determinacy of smooth map-germs, Bull. London Math Society 13 (1981), 481-539. 